\title{
Indagini preliminari e rilievo architettonico di due magazzini sull'antico sito di Thamugadi (Timgad)
}

Francesco Martorella e Fatima-Zohra Bahloul

\section{(2) OpenEdition}

\section{Journals}

Edizione digitale

URL: http://journals.openedition.org/cefr/4092

DOI: $10.4000 /$ cefr.4092

ISSN: 2282-5703

Editore

École française de Rome

Notizia bibliografica digitale

Francesco Martorella e Fatima-Zohra Bahloul, « Indagini preliminari e rilievo architettonico

di due magazzini sull'antico sito di Thamugadi (Timgad) », Chronique des activités archéologiques de

l'École française de Rome [Online], Maghreb, Messo online il 24 février 2020, consultato il 26 février

2020. URL : http://journals.openedition.org/cefr/4092 ; DOI : 10.4000/cefr.4092

Questo documento è stato generato automaticamente il 26 febbraio 2020.

(c) École française de Rome 


\title{
Indagini preliminari e rilievo architettonico di due magazzini sull'antico sito di Thamugadi (Timgad)
}

\author{
Francesco Martorella e Fatima-Zohra Bahloul
}

\section{NOTE DELL'AUTORE}

Le attività di ricerca e di formazione si sono svolte dal 20 ottobre al 28 ottobre 2016 nell'ambito dell'accordo quadro tra l'École française de Rome e l'université de Batna 1 (Institut d'Architecture et d'Urbanisme). A tal proposito si ringraziano la Prof. Catherine Virlouvet, già Direttrice École française de Rome, e il Prof. Abdeslam Dif, Rettore dell'université de Batna 1. I risultati preliminari della cooperazione sono stati presentati da Fatima-Zohra Bahloul e da Francesco Martorella nel corso della conferenza tenutasi giovedì 27 ottobre 2016 presso l'Institut d'Architecture et d'Urbanisme (université de Batna 1). Le attività sul campo sono state coordinate da Francesco Martorella.

Hanno partecipato alla fase formativa studenti del Corso di Architettura e di archeologia dell'université de Batna 1 e tecnici della Conservazione del Ministère de la culture $^{1}$. Aiuto prezioso alla logistica si deve al dr. Madjid Belkares, gestionnaire chez OGEBC (Office National de Gestion et Exploitation des Biens Culturels Protégés) du site archéologique de Timgad ${ }^{2}$.

\section{Situation des deux entrepôts étudiés}

1 Timgad, l'antique Thamugadi, est située aux pieds des monts des Aurès, sur la route qui relie Lambaesis (Lambèse) et Mascula (Khenchela). La colonie fut fondée en l'an 100 
apr. J.-C., sur l'ordre de Trajan. Son plan en damier fut tracé par les ingénieurs de la III légion Auguste. La ville est structurée par deux voies importantes bordées de portiques, d'est en ouest, le decumanus maximus et, du nord au sud, le cardo maximus. Des voies secondaires et parallèles au decumanus et au cardo, forment des îlots d'à peu près $20 \mathrm{~m}$ de côté.

2 Nous nous intéressons à deux entrepôts, aujourd'hui en majeur partie détruits, ils étaient construits sur deux caves, occupant deux îlots qui donnaient sur le portique sud du decumanus maximus. L'un était situé à l'extérieur de l'enceinte de la ville primitive, juste à côté du grand arc dit de Trajan, et l'autre était placé à l'ouest du forum (fig. 1-2 ).

Fig. 1 - Ville de Thamugadi (Timgad) avec indication en bleu des entrepôts A et B.

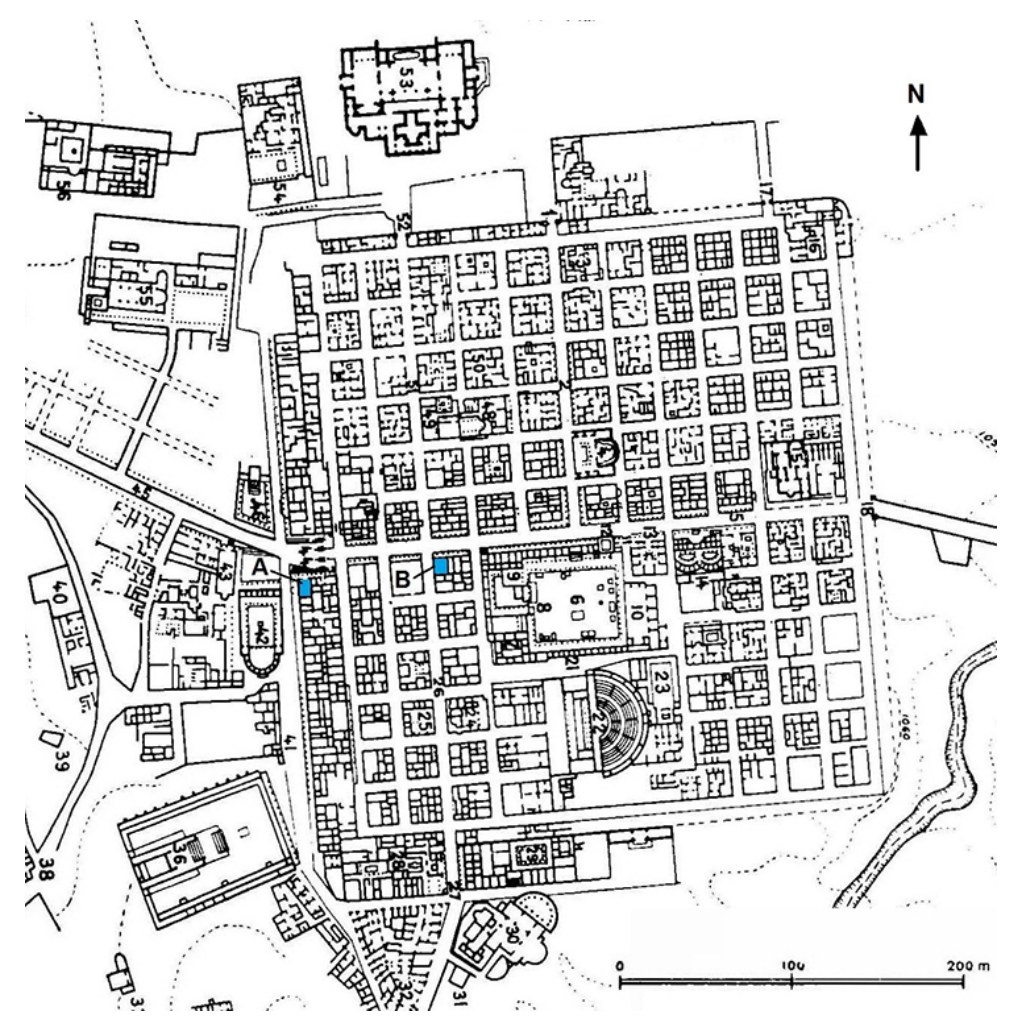

D'après Courtois 1951 (réélaboré par F.-Z. Bahloul). 
Fig. 2 - Thamugadi (Timgad). Photo satellite de la partie ouest de la ville avec l'indication des caves $A$ et $B$.

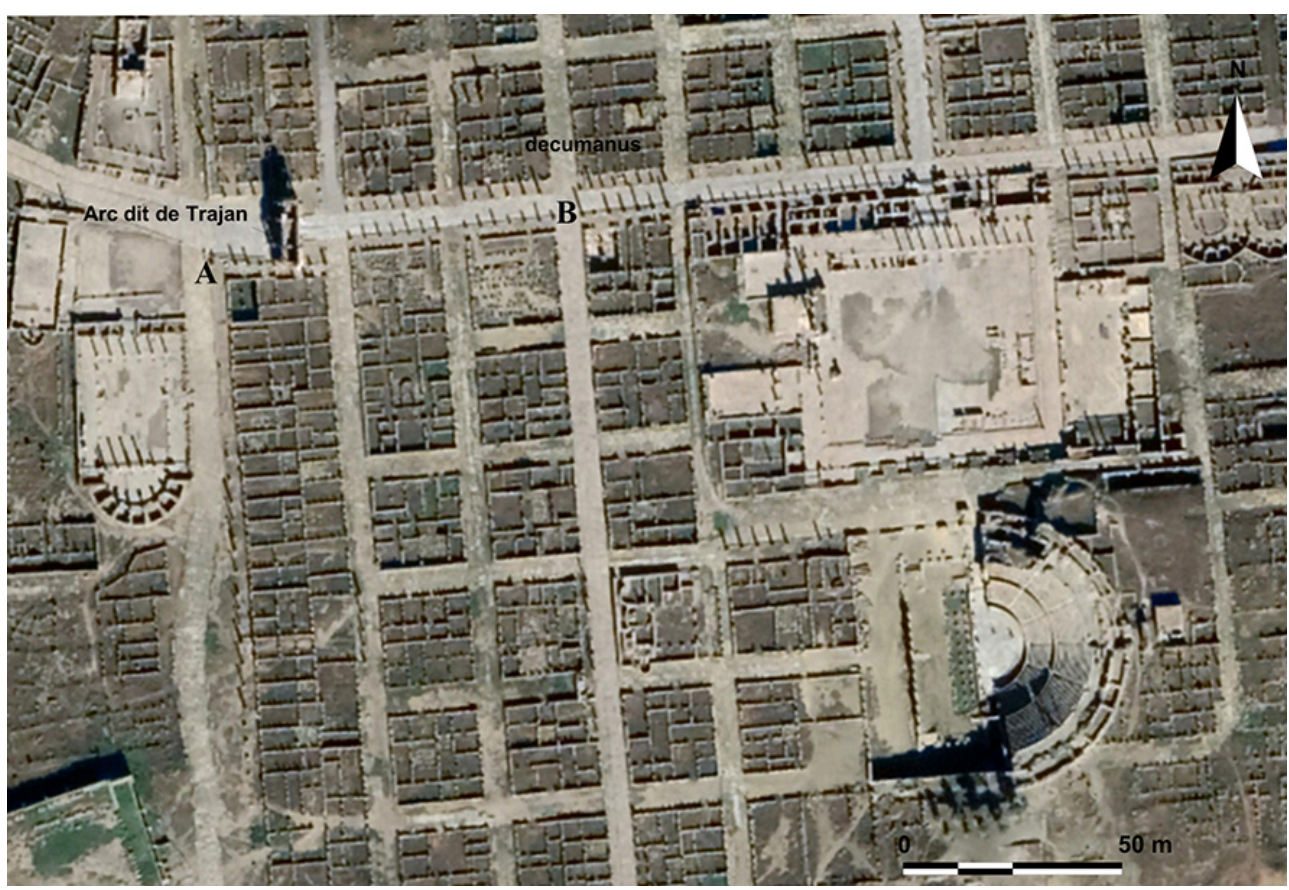

(c) Google Earth, réélaboré par F.-Z. Bahloul.

3 Le premier îlot où était installé l'entrepôt $\mathrm{A}$ n'était pas de forme proprement carrée comme ceux de la ville primitive, mais constituait plutôt un grand quartier rectangulaire qui faisait partie de toute une bande de constructions tardives et qui étaient placées de part et d'autre de l'enceinte primitive ouest de la ville. L'entrepôt A a été dégagé dans les années 1930 et un bref compte rendu a été publié dans les rapports de fouilles de M. Christofle ${ }^{3}$ Cet entrepôt est situé à l'angle nord-ouest du long quartier en question. Plusieurs salles de différentes dimensions se trouvent sur les côtés sud et est de l'entrepôt. Ces pièces que nous numérotons a1, a2, etc., étaient largement ouvertes sur les voies, et il est fort probable qu'elles étaient destinées au commerce (fig. 3-4).

4 Les murs de la cave A sont construits en opus africanum. À la hauteur des parties basses d'origine, la maçonnerie entre les harpes est construite de moellons informes et de différentes tailles, ressemblant à ceux des restes encore visibles de la muraille ouest. Il est possible que les matériaux aient été récupérés lors de la destruction de cette dernière lors de l'extension de la ville primitive, puis remployés au niveau de la cave.

5 Le rapport de Charles Godet, inspecteur du chantier de fouilles, signé par M. Christofle, l'architecte en chef des monuments historiques, fait état de plusieurs objets retrouvés dans la cave A : trois lampes chrétiennes, une petite tirelire en terre cuite, une petite clé en bronze reliée à l'anneau d'un coffret, deux crochets de balances romaines, une boucle en bronze avec son ardillon, une fibule en bronze, elle aussi avec son ardillon, restes de cuillers en bronze, une grosse bague, trois anneaux en bronze de différents diamètres, un lot de monnaies en bronze, 13 épingles à cheveux complètes et 63 fragments en os et en ivoire, une plaque rectangulaire en os, un fragment de bracelet, des boutons, de nombreux fragments de vases en terre en verre et enfin une borne milliaire. 
6 À part les parures féminines, on retrouve surtout des objets qui témoignent que ces espaces étaient destinés au commerce et au dépôt des marchandises d'autant plus que le marché de Sertius leur fait face de l'autre côté de la voie du capitole.

Fig. 3 - Cave qui supportait l'entrepôt A. Revêtement intérieur du mur périphérique ouest.

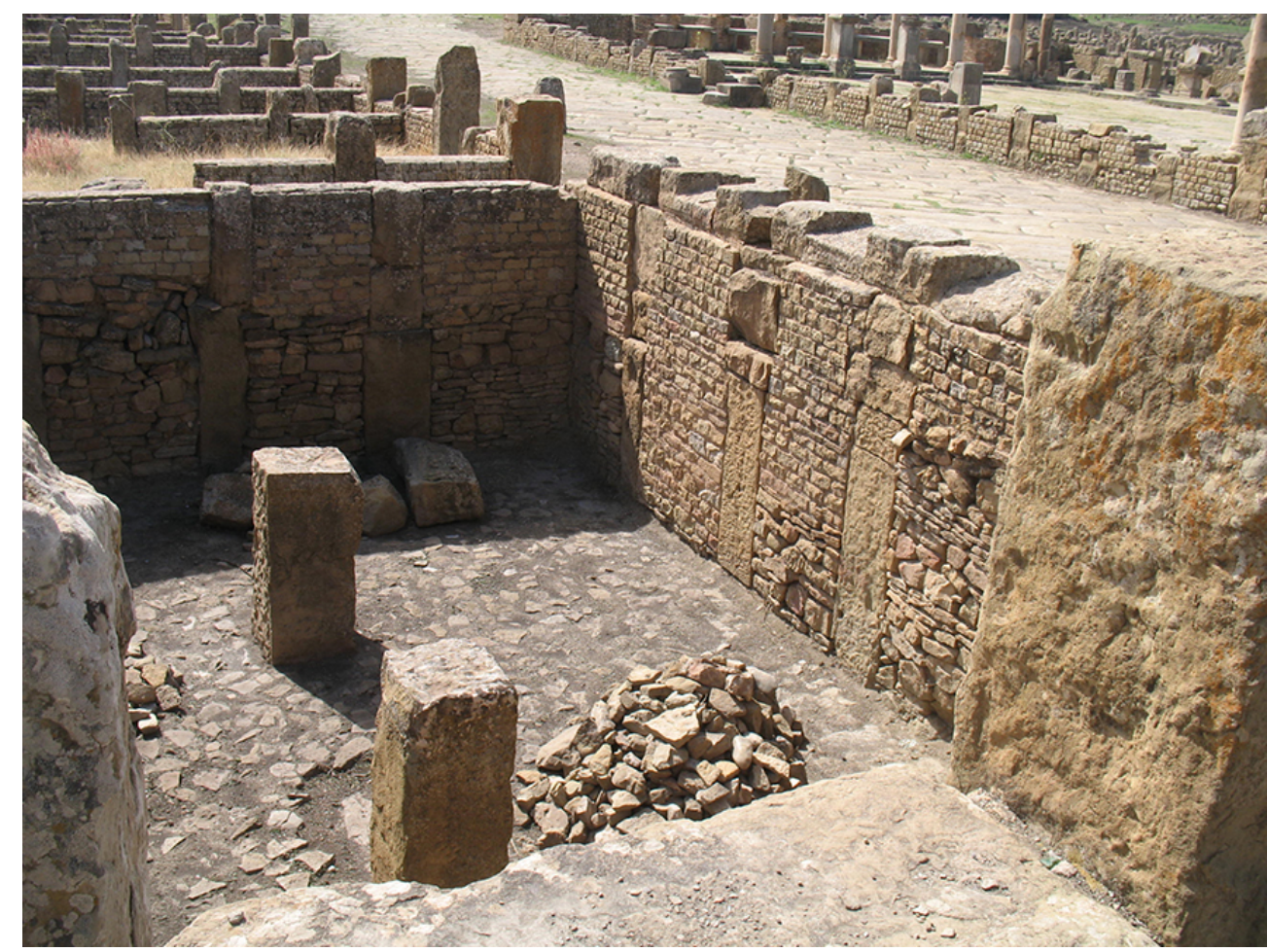

F.-Z. Bahloul. 
Fig. 4 - Situation de l'entrepôt A dans grand quartier rectangulaire.

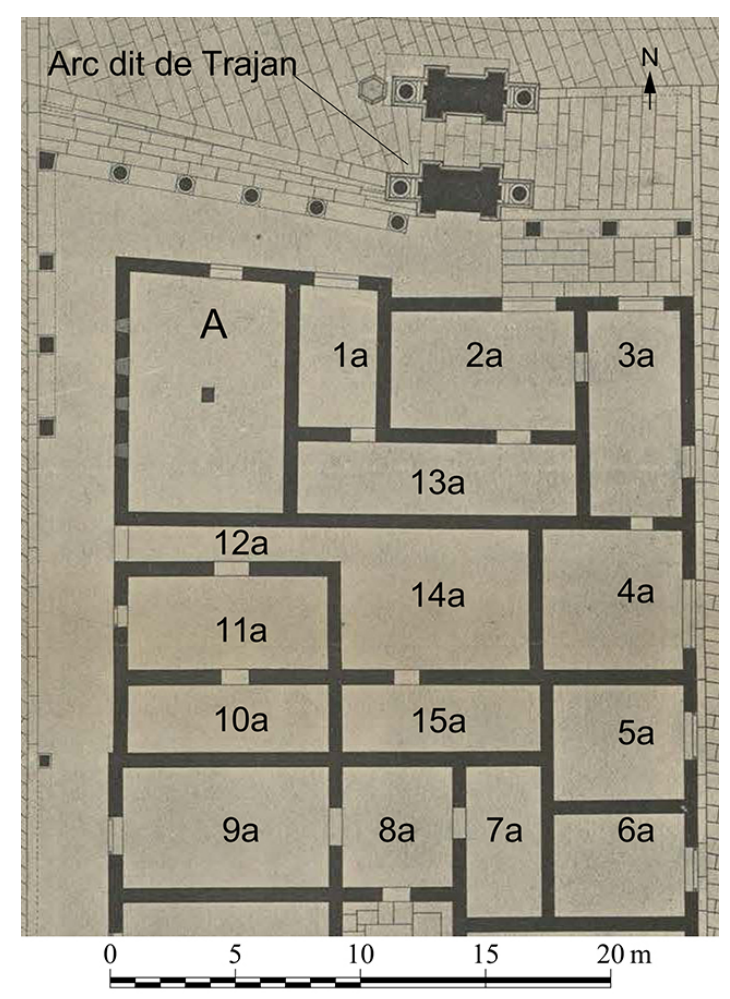

D’après Ballu 1902, pl. VII, réélaboré par F.-Z. Bahloul.

7 Le second îlot, se trouvant à l'intérieur de la ville primitive, a été dégagé en $1901^{4}$; ce n'est que cinq ans plus tard qu'une description paraitra, n'occupant que deux pages de l'ouvrage de Boeswillwald'5. L'entrepôt B occupait l'angle nord-ouest de cet îlot: une grande maison dont la façade nord donnait directement sur le portique du decumanus. Juste à côté, un long couloir (1) débouchait sur une petite pièce (11), espace de distribution avec la cour centrale (12) garnie d'un bassin semi-circulaire et une grande pièce située au sud-ouest 9 . Entre cette dernière pièce et l'entrepôt $B$ fut aménagé un autre petit magasin (10) ouvert sur la voie perpendiculaire au decumanus. Deux autres pièces $(2,3)$ s'alignant avec le couloir et ouvertes, elles aussi, sur le portique du decumanus, sont considérées comme des magasins. La salle 3 communique avec une autre grande salle (4). L'entrée de la maison se faisait par l'arrière, sur la voie parallèle au decumanus. On accédait par un escalier au vestibule (8) qui communiquait en face directement avec la cour centrale 12 et à l'est avec deux pièces ( 6 et 7) qui conservent encore leurs hypocaustes en place (fig. 5-6).

Les salles (6 et 7) avaient été identifiées comme des bains ${ }^{6}$, elles furent reconnues plus tard comme seulement des salles chauffées ${ }^{7}$. Il n'y a aucune trace de conduite d'eau ou d'évacuation ni aucune trace d'enduit hydraulique. La partie où se trouve une ouverture arquée près du sol de la salle 6 est une restauration moderne et tout autour il n'y a aucune trace de chaufferie. Il semble que cette ouverture servait plutôt à l'aération. Les intérieurs des murs des deux petites salles sont doublés d'un muret, haut de $60 \mathrm{~cm}$, construit en briques; la partie supérieure de ces murets de soubassement comporte des logements de poutres en bois qui supportaient le plancher. Il me semble que nous avons là des salles entrepôts, espaces réservés pour la bonne conservation de 
la nourriture, en relation avec les autres dépôts et boutiques de commerce de l'ensemble du bâtiment.

Fig. 5 - Situation de l'entrepôt B dans l'îlot carré.

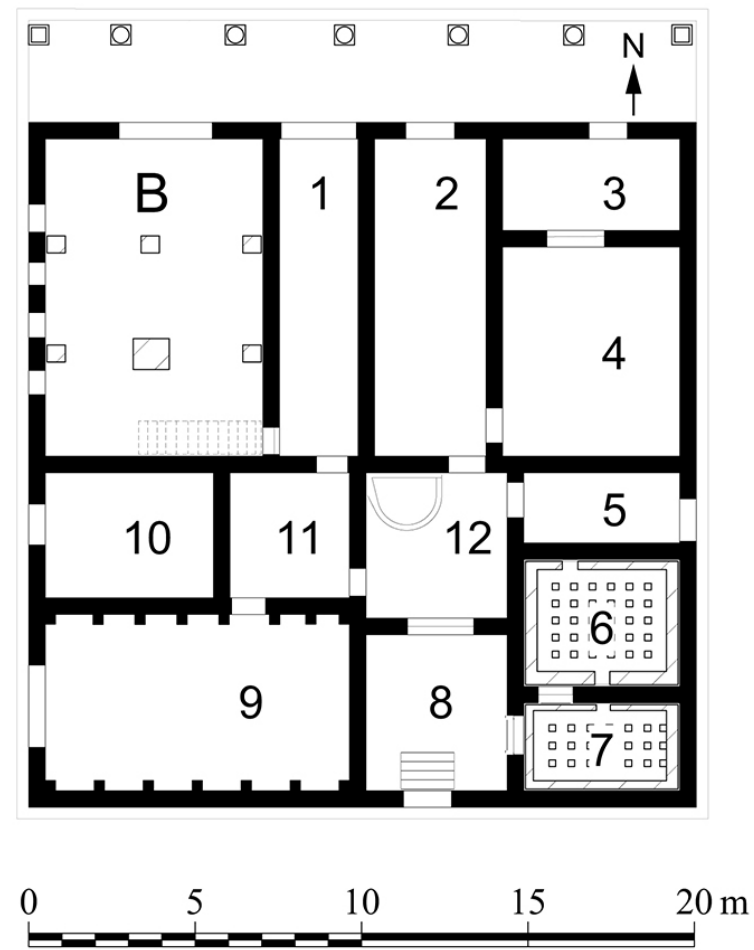

D’après Boeswillwald et al. 1905, p. 337, fig. 166, réélaboré par F.-Z. Bahloul. 
Fig. 6 - Cave B, Parement extérieur du mur ouest.

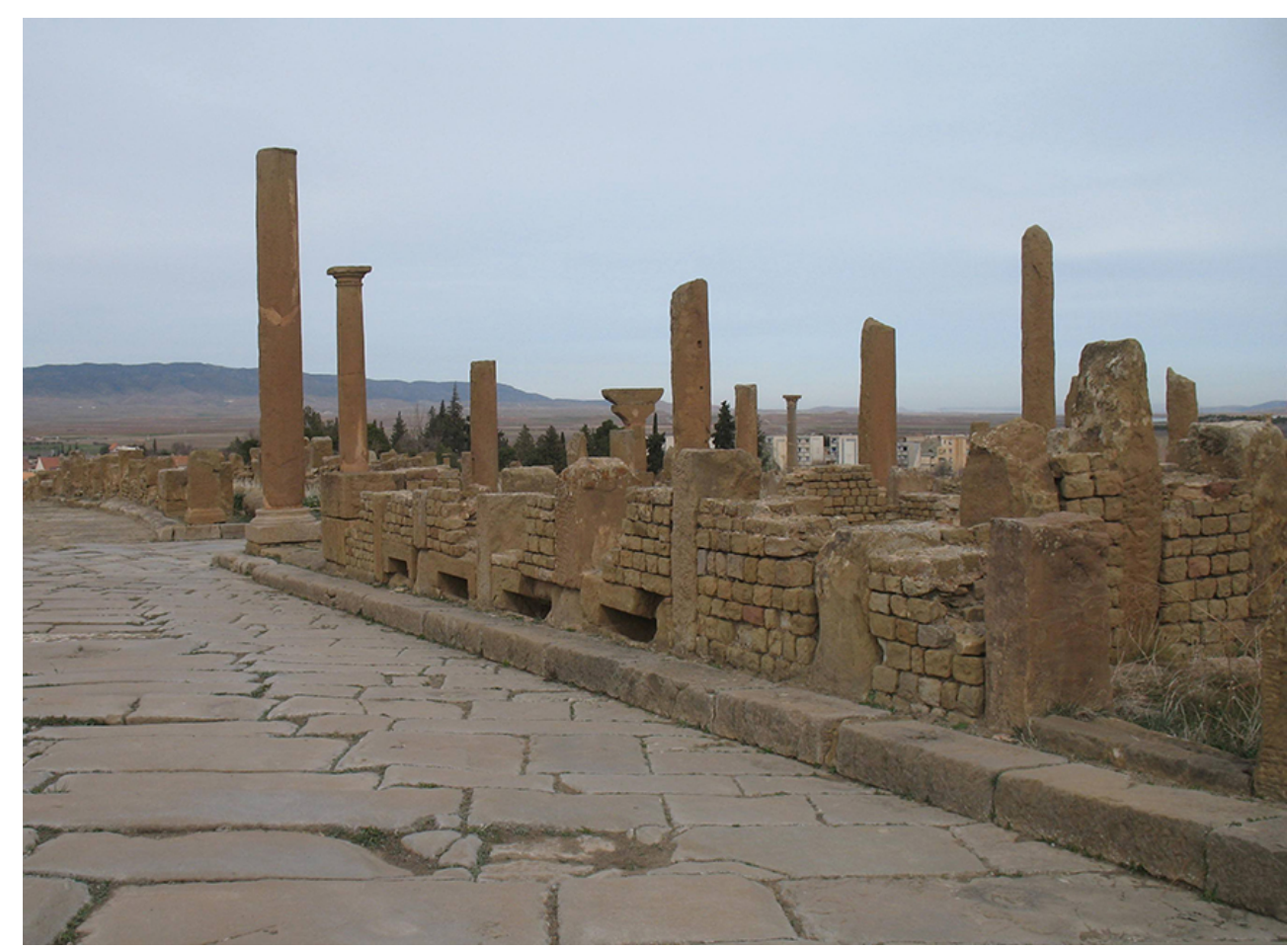

F.-Z. Bahloul.

9 Si Timgad n'a livré aucune inscription mentionnant des horrea, à l'inverse de Djemila, par contre, il y existe plusieurs autres bâtiments, relativement bien conservés et possédant certaines caractéristiques des entrepôts. Il est du reste possible que d'autres entrepôts soient encore enfouis, puisque le site de Timgad n'est pas entièrement fouillé. Nous pouvons approfondir la recherche sur d'autres entrepôts, toujours à Timgad, et même sur d'autres monuments, d'autant plus que la ville était dotée de trois marchés. En étudiant tous ces témoignages, il est envisageable de retracer l'activité économique de la ville, voire de la région.

\section{F.-Z. Bahloul ${ }^{8}$}

\section{Metodologia}

\section{Premessa}

Il progresso tecnologico, la pianificazione degli interventi, l'ottimizzazione delle risorse unite alle problematiche della tutela e della valorizzazione dei siti archeologici coinvolgono oggi un ampio e variegato spettro di discipline e metodologie, alcune specifiche, altre mutuate da altri settori di ricerca; tra queste, le rappresentazioni grafiche in formati digitali, le nuove tecniche di rilievo e di diagnostica, la gestione informatica e la realtà virtuale. La tecnologia laser scanning, nata in ambito ambientale e ingegneristico, ha trovato ampio utilizzo nel campo dei beni monumentali, nel settore della ricerca, della salvaguardia e della valorizzazione del patrimonio.

11 Il laser scanner, detto anche laser 3D, è uno strumento che consente il rilevamento di modelli tridimensionali di oggetti a scale e risoluzioni differenti, acquisendo 
velocemente e in modo automatico una grande quantità di dati. La scansione tridimensionale di innumerevoli punti con coordinate $x, y, z$ (il cui insieme costituisce le cosiddette "nuvole" di punti) consente, attraverso l'acquisizione di una o più nuvole, la creazione e l'interrogare del modello digitale, ottenendo svariate informazioni (animazione 3D, solid image, calcolo dei volumi, curve di livello, quote, piante, prospetti, sezioni 2D e 3D, ortofoto, ecc.).

Le deformazioni, le linee di discontinuità e le zone d'ombra presenti sull'oggetto vengono corrette attraverso l'interpolazione di punti acquisiti da più stazioni di scansione. Lo strumento è in grado, inoltre, di riconoscere i vari tipi di materiali in funzione della loro riflettanza e, con l'ausilio di una camera digitale associata, assegnare il colore a ogni singolo punto. I dati acquisiti vengono applicati sul modello tridimensionale grazie a un processo di parametrizzazione e di texture mapping.

A Timgad, le attività di rilievo architettonico dei magazzini A e B sono state eseguite con l'ausilio della tecnologia laser scanning. I rilievi in ambiente outdoor sono stati realizzati con laser Scanner Faro 3D ad alta velocità, unendo tecnologia di scansione ed elevata precisione (fig. 7, tab. 1$)^{9}$.

Fig. 7 - Area del magazzino B. Laser scanner Faro 3D in fase di pre-rilievo automatizzato.

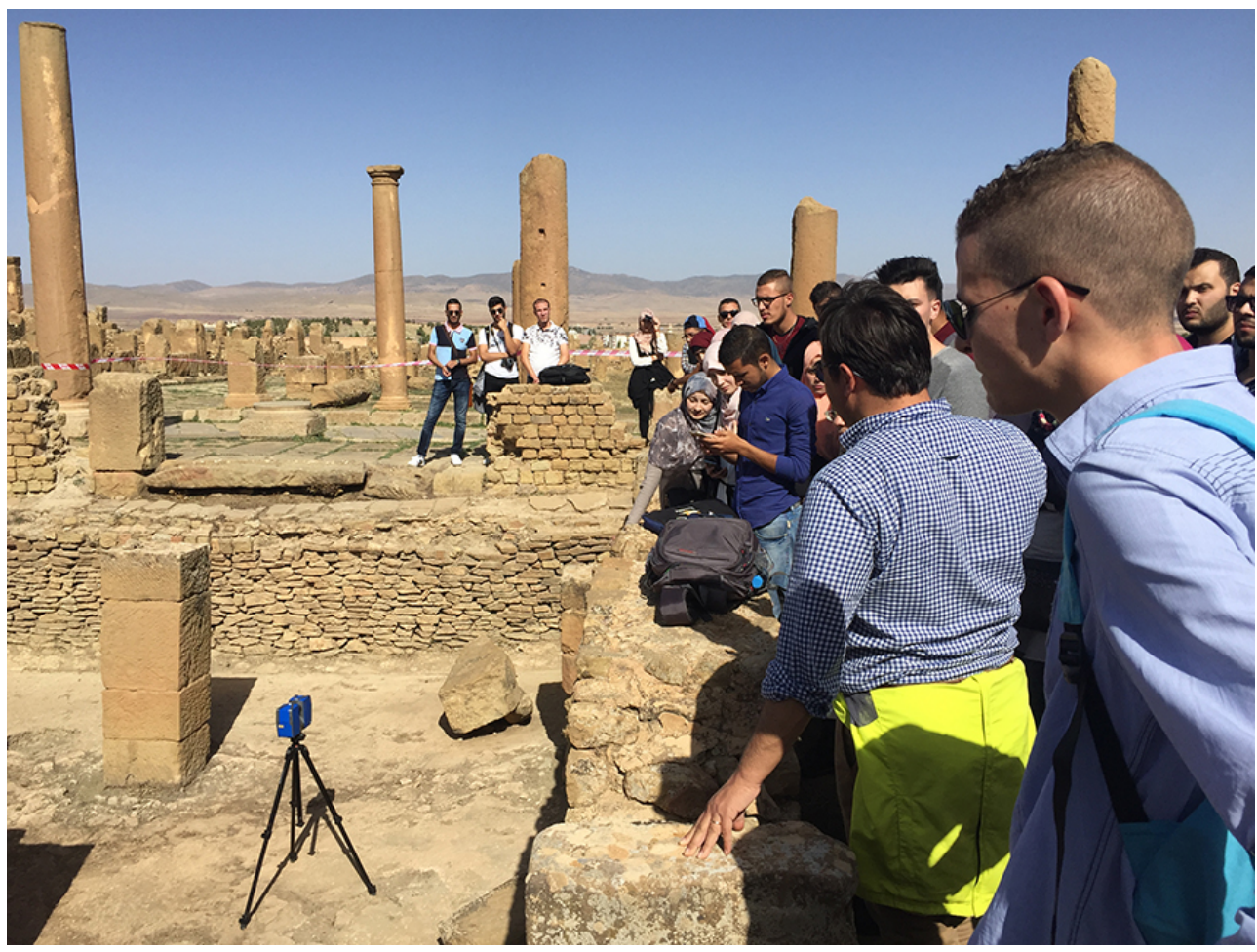

Fr. Martorella. 
Tab. 1 - Specifiche di prestazione del Laser Scanner Faro 3D.

\begin{tabular}{|c|c|c|c|c|}
\hline \multicolumn{2}{|c|}{$\begin{array}{l}\text { Ranging unit } \\
\text { Unambiguity interval: } \\
\text { Range: } \\
\text { Velocità di misura (punti/sec.): } \\
\text { Errore di distanza lineare': }\end{array}$} & \multicolumn{3}{|c|}{$\begin{array}{l}\text { da } 122 \text { fino a } 488 \mathrm{kpts} / \mathrm{sec} .: 614 \mathrm{~m} \text {; a } 976 \mathrm{kpts} / \mathrm{sec} .: 307 \mathrm{~m} \\
0,6 \mathrm{~m} \text { - } 130 \mathrm{~m} \text { indoor o outdoor con incidenza verticale su superficie riflettente (90\%) } \\
122,000 / 244,000 / 488,000 / 976,000 \\
\pm 2 \mathrm{~mm}\end{array}$} \\
\hline Rumore $^{2}$ & (10 $\mathrm{m}$ & (910 m- filtrato ${ }^{3}$ & @ $25 \mathrm{~m}$ & @25m-filtrato ${ }^{3}$ \\
\hline (9) $90 \%$ refl. & $0,3 \mathrm{~mm}$ & $0.15 \mathrm{~mm}$ & $0,3 \mathrm{~mm}$ & $0.15 \mathrm{~mm}$ \\
\hline (@) 10\% refl. & $0.4 \mathrm{~mm}$ & $0.2 \mathrm{~mm}$ & $0.5 \mathrm{~mm}$ & $0.25 \mathrm{~mm}$ \\
\hline \multicolumn{2}{|l|}{$\begin{array}{l}\text { Risoluzione: } \\
\text { HDR: } \\
\text { Parallasse: }\end{array}$} & \multicolumn{3}{|c|}{$\begin{array}{l}\text { fino a } 165 \text { megapixel a colori } \\
\text { registrazione immagine High Dynamic Range (HDR), 3x / 5x } \\
\text { design co-assiale }\end{array}$} \\
\hline \multicolumn{2}{|c|}{$\begin{array}{l}\text { Unifa di defiessione } \\
\text { Campo di vista (vert./orizz.): } \\
\text { Risoluzione (vert./oriz.): } \\
\text { Max. velocità di scansione vert.: }\end{array}$} & \multicolumn{3}{|c|}{$\begin{array}{l}300^{\circ} / 360^{\circ} \\
0.009^{\circ}\left(40.9603 \mathrm{D} \text {-pixel a } 360^{\circ}\right) / 0.009^{\circ}\left(40.960 \text { 3D-pixel a } 360^{\circ}\right) \\
5.820 \mathrm{rpm} 097 \mathrm{~Hz}\end{array}$} \\
\hline \multicolumn{2}{|c|}{$\begin{array}{l}\text { Classe laser: } \\
\text { Lunghezza d'onda: } \\
\text { Divergenza del raggio: } \\
\text { Diametro del raggio in uscita: }\end{array}$} & \multicolumn{3}{|c|}{$\begin{array}{l}\text { laser classe } 1 \\
1550 \mathrm{~nm} \\
\text { Typical } 0,19 \mathrm{mrad}\left(0,011^{\circ}\right)(1 / \mathrm{e}, \text { halfangle) } \\
\text { Typical } 2,25 \mathrm{~mm}(1 / \mathrm{e})\end{array}$} \\
\hline \multicolumn{2}{|c|}{$\begin{array}{l}\text { Gestione dati e controllo } \\
\text { Memorizazione dei dati: } \\
\text { Controllo dello scanner: } \\
\text { Accesso WLAN: }\end{array}$} & \multicolumn{3}{|c|}{$\begin{array}{l}\text { SD, SDHCTM, SDXCTM; scheda da } 32 \text { GB inclusa nella fornitura } \\
\text { tramite touchscreen e WLAN } \\
\text { il controllo e la visualizazione delle scansioni a distanza sono possibili su dispositivi mobile con Flash }{ }^{\circ} \text { e HTML5. }\end{array}$} \\
\hline \multicolumn{2}{|c|}{$\begin{array}{l}\text { Compensatore biassiale: } \\
\text { Sensore di altezza: }\end{array}$} & \multicolumn{3}{|c|}{$\begin{array}{l}\text { fornisce per ogni scansione informazioni relative al livello; precisione } 0,015^{\circ} \text {; range di misurazione } \pm 5^{\circ} \\
\text { grazie ad un barometro elettronico è possibile determinare per ogni scansione l'altezza relativa rispetto a un } \\
\text { punto di riferimento } \\
\text { la bussola elettronica individua l'orientamento della scansione. Ė inoltre disponibile una funzione di calibrazione } \\
\text { ricevitore GPS integrato }\end{array}$} \\
\hline
\end{tabular}

Il dispositivo, che consente di realizzare scansioni architettoniche fino a $130 \mathrm{~m}$ di distanza e di visualizzare in tempo reale i dati registrati, ha garantito un'elevata affidabilità e precisione del rilievo. Nella registrazione delle immagini la funzionalità HDR (High Dynamic Range) del Laser scanner Faro 3D ha permesso di risolvere condizioni di illuminazione differenti, uniformando la qualità delle immagini in ambiente molto luminoso e in ombra. Il ricevitore GPS integrato ha consentito di correlare le singole scansioni in fase di post-elaborazione; i dati di scansione 3D sono stati importati ed elaborati in fase post-processing.

\section{Rilievo architettonico ed elaborazione dei dati}

Dopo una pulizia delle aree oggetto di indagine (magazzini A e B) e secondo gli approcci metodologici centrati principalmente sull'archeologia della costruzione e il rilievo architettonico, lo studio si è focalizzato sulle strutture murarie, elaborando un sistema unico di georeferenziazione. Le attività di rilievo ed elaborazione dei dati sono state divise in tre fasi in collaborazione con specialisti di differenti discipline (architettura, archeologia, ingegneria applicata all'uso delle nuove tecnologie) abili a integrare competenze e risorse tecniche. Le attività sono state completate da lezioni teoriche sulla metodologia da applicare nelle attività di rilievo, con momenti di visualizzazione dei dati ed esercitazioni sull'uso della strumentazione in ambiente outdoor (fig. 8). 
Fig. 8 - Attività formative di rilievo architettonico.

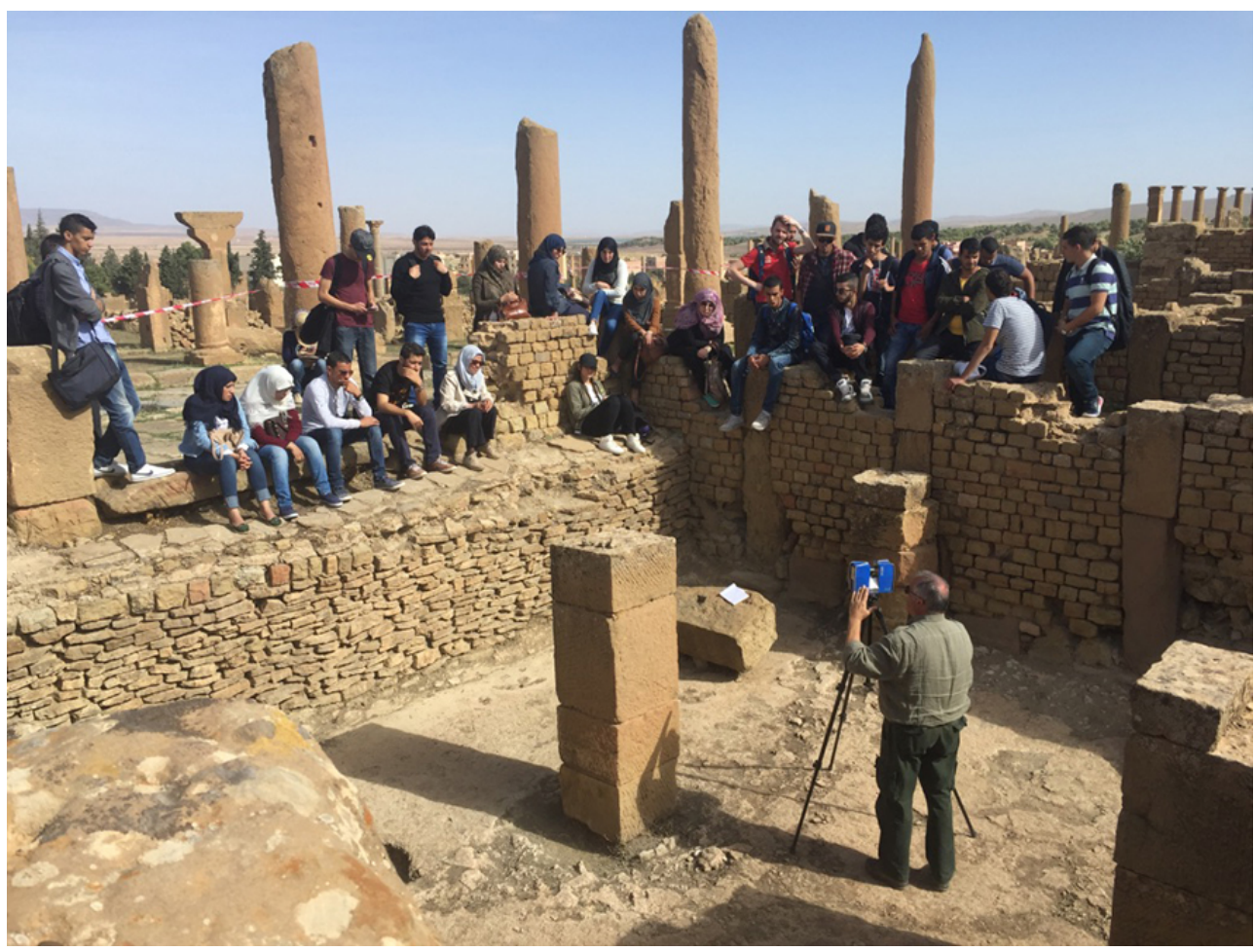

Fr. Martorella.

\section{Fase 1 - acquisizione dati in ambiente outdoor}

Lo scanner 3D è stato trasportato nell'area dei magazzini da rilevare utilizzando la valigia in dotazione con batterie e cavi di collegamento; montato su un treppiede dotato di livella a bolla, lo strumento è stato posizionato e collegato alle batterie. Attraverso uno schermo touch, lo scanner è stato programmato, impostando parametri di alta risoluzione e precisione: maggior numero di punti acquisiti, migliore dettaglio della scansione. Impostati i parametri, lo scanner ha iniziato a emettere il raggio laser colpendo la parte di oggetto rientrante nel suo campo visivo. Ciascuna scansione ha prodotto una nuvola di punti (fig. 9). Alla fine di ogni scansione, i dati sono stati registrati su una scheda di memoria SD. 
Fig. 9 - Nuvole di punti su immagine HDR del prospetto del muro perimetrale ovest (magazzino A; particolare).

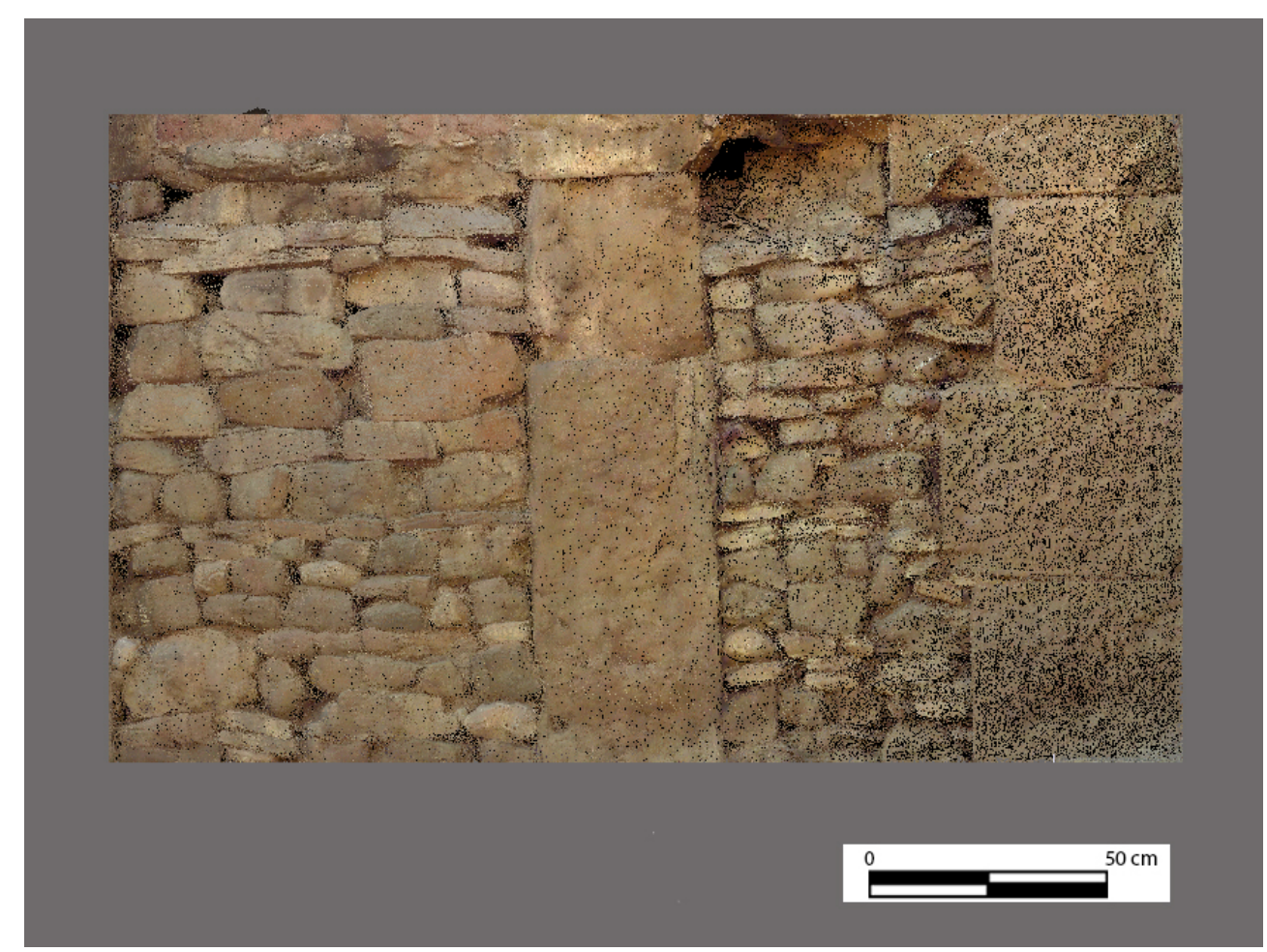

Fr. Martorella.

\section{Fase 2 - processamento dei dati con software di base}

17 Alla fine di ogni scansione, il software di base, chiamato programma di "proprietà" ha permesso di codificare ed esportare i dati recepiti dallo scanner. Il prodotto della scansione è costituito da nuvole di punti ad altissima densità che descrivono con estrema esattezza la superficie dell'oggetto unitamente a quanto compare nel campo di vista strumentale. Tale prodotto, molto lontano dalle classiche rappresentazioni grafiche realizzate con il metodo del rilevamento diretto, ha reso necessari il "filtro" e la sintesi soggettiva del rilevatore in grado di selezionare, discretizzare e codificare gli elementi di interesse (fig. 10). 
Fig. 10 - Prospetto del muro perimetrale ovest (magazzino A). Insieme di nuvole di punti ad altissima densità sovrapposte a immagini ad alta definizione (rilievo fotogrammetrico).

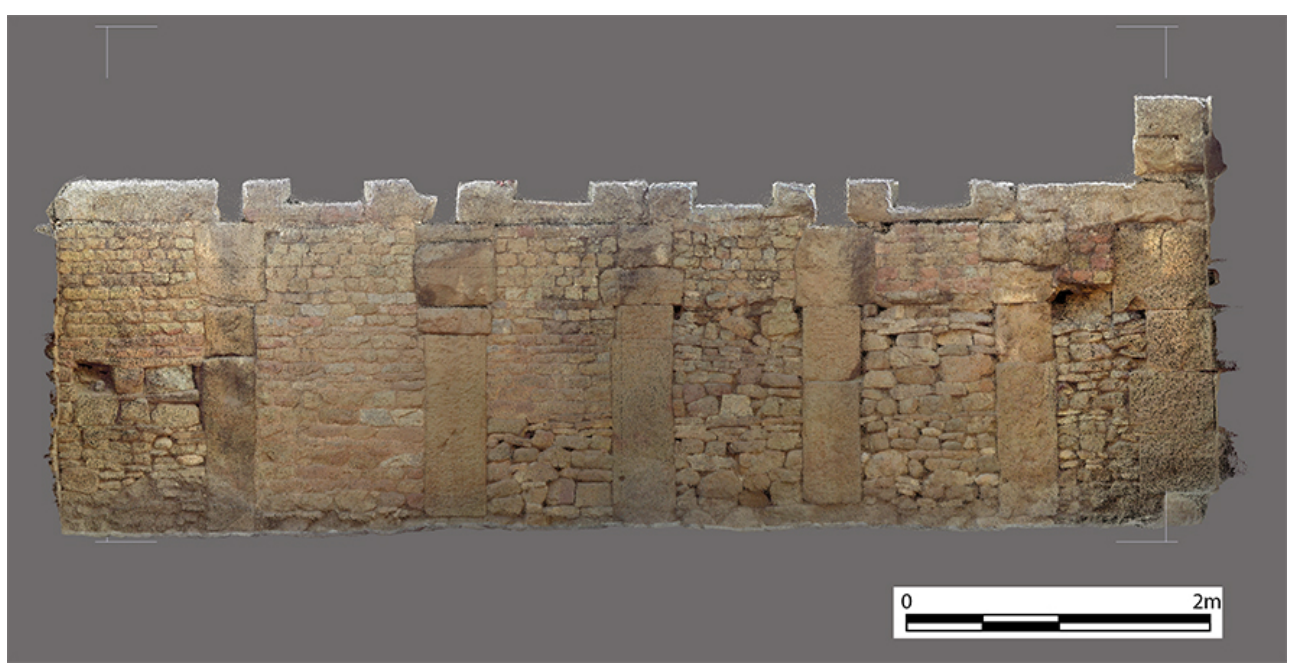

Fr. Martorella.

\section{Fase 3 - processamento dei dati con software di modellazione}

L'enorme banca dati prodotta dal rilievo digitale è stata trattata attraverso un software di modellazione capace di intervenire in maniera mirata e discriminante. I punti delle scansioni, in una prima fase fornite in un sistema di riferimento cartesiano, sono stati appositamente georeferenziati in termini assoluti e relativi. Un software di disegno CAD 3D ha consentito di produrre gli elaborati finali (fig. 11).

Fig. 11 - Schermata di visualizzazione 3D del magazzino A con software di disegno CAD.

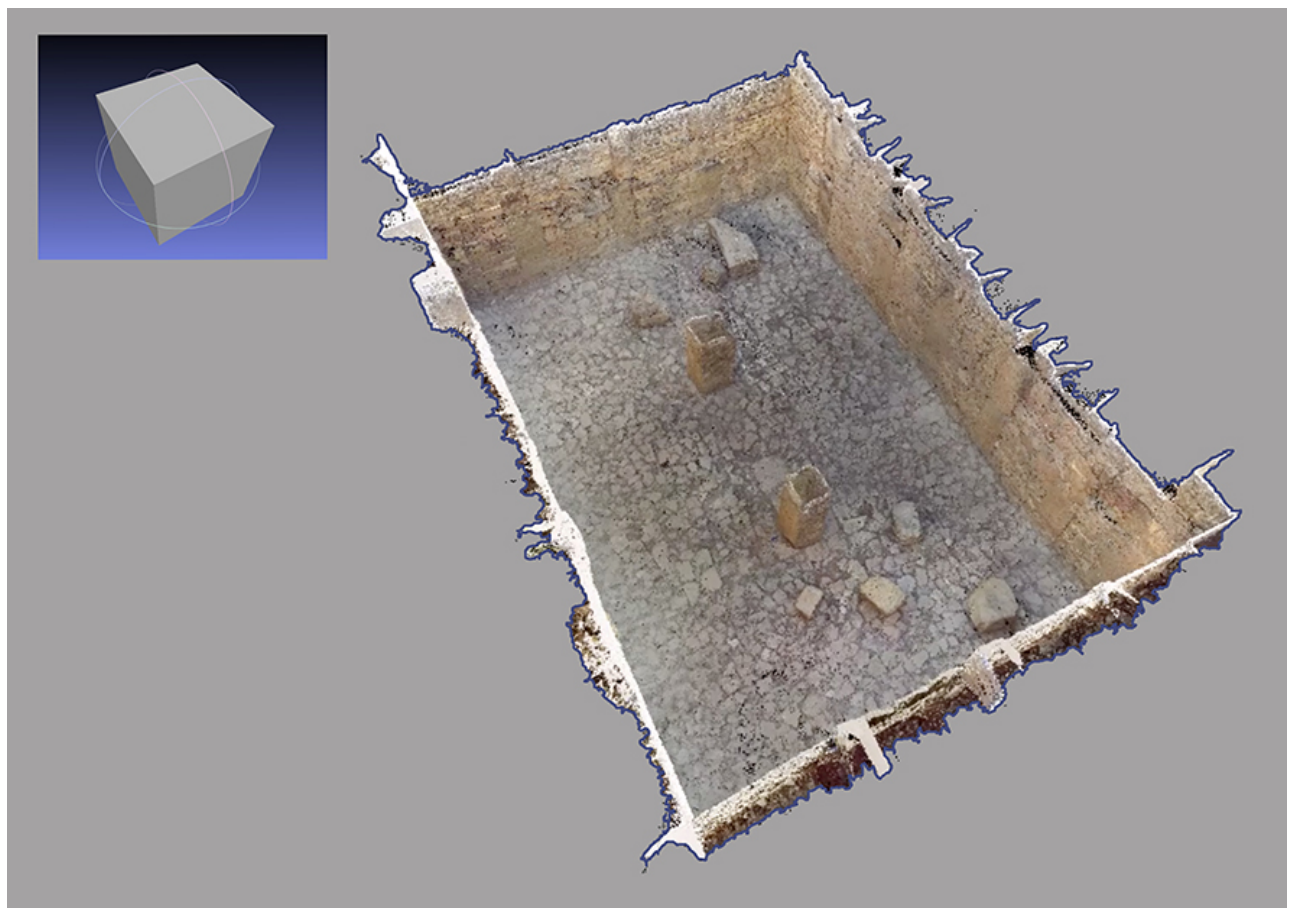

Fr. Martorella. 
19 In questa fase, l'attenzione si è focalizzata su un'attenta pianificazione del rilievo architettonico attraverso un imprescindibile inquadramento topografico. Il sistema di riferimento materializzato da punti stabili e facilmente riconoscibili è risultato un nodo fondamentale per la riuscita di tutte le operazioni di rilievo e di integrazione dei dati e dei risultati.

\section{Analisi planimetrica}

\section{Magazzino A}

Il magazzino A è una struttura rettangolare di $85 \mathrm{~m}^{2}$, di cui $60 \mathrm{~m}^{2}$ calpestabili, realizzata come ambiente unico e indipendente dai vani che lo fiancheggiano. Lo spessore dei muri perimetrali nord, sud, est e ovest, realizzati in opus africanum, è di $0,50 \mathrm{~m}$. L'ingresso del magazzino è sul lato nord lungo il decumanus, in corrispondenza del portico colonnato. La soglia d'ingresso con apertura verso l'interno presenta una larghezza di $1,20 \mathrm{~m}$ e uno spessore di 0,50 m (fig. 12). In posizione assiale sono ancora in situ due pilastri in pietra quadrata dello spessore di $0,50 \mathrm{~m}$ con altezza massima di $1,21 \mathrm{~m}$ (pilastro sud) e 1,39 m (pilastro nord).

Fig. 12 - Magazzino A.

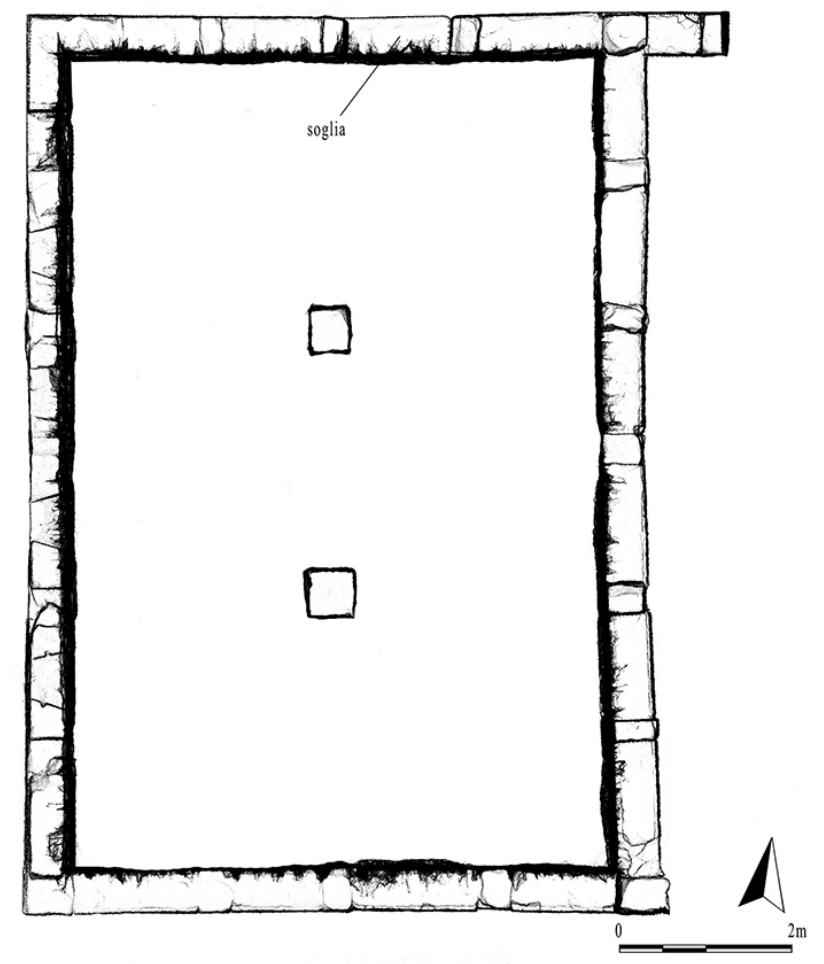

Fr. Martorella.

21 L'edificio è stato interamente scavato dalle missioni francesi che hanno restaurato gran parte della parte sommitale dei paramenti dei muri perimetrali. La pulizia e il diserbo che hanno preceduto il rilievo delle strutture hanno evidenziato un piano pavimentale costituito da pietre di medie dimensioni (0,20-0,40 m) (fig. 13). 
Fig. 13 - Magazzino A. Piano pavimentale con restituzione fotogrammetrica.

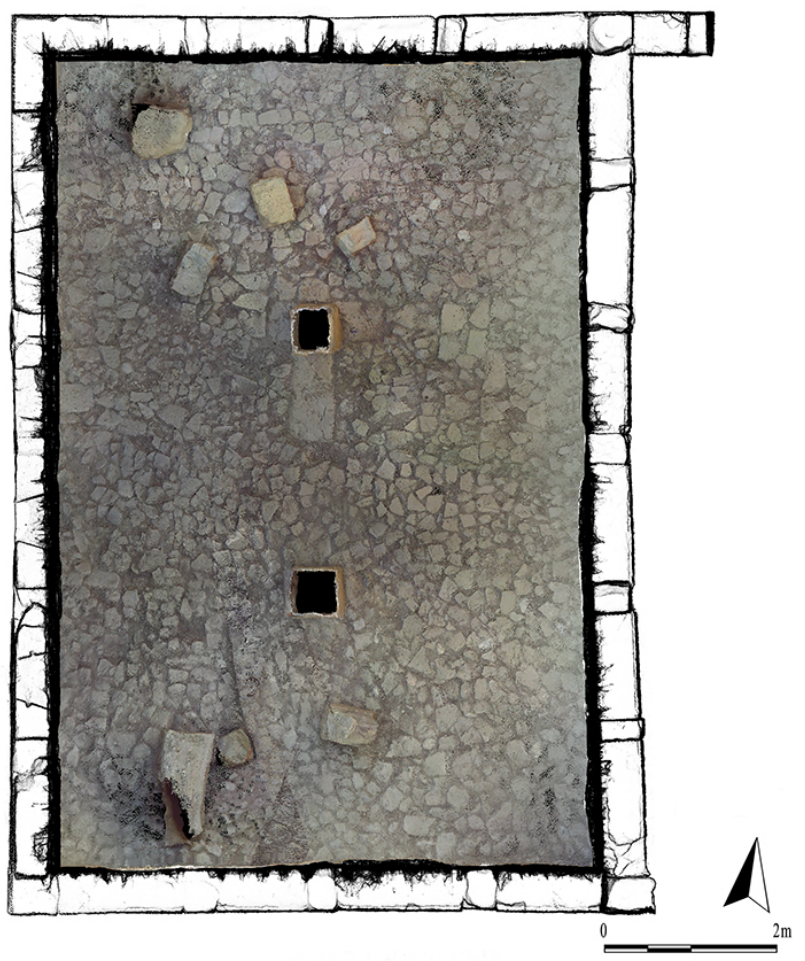

Fr. Martorella.

Il muro perimetrale nord si caratterizza per una lunghezza di 8,07 $\mathrm{m}$ e un'altezza massima conservata di $3,72 \mathrm{~m}$ (altezza calcolata dal piano pavimentale). Il prospetto interno del muro perimetrale nord evidenzia una soglia d'ingresso non perfettamente centrata e allineata con i pilastri interni (fig. 14). Il muro perimetrale est presenta una lunghezza di 10,43 m (paramento esterno) e di 9,50 m (paramento interno; fig. 15) e costituisce, nello stesso tempo, il limite ovest di un vano adiacente. La lunghezza del muro perimetrale sud è di 7,44 $\mathrm{m}$ (paramento esterno) e di 6,37 $\mathrm{m}$ (paramento interno; fig. 16). Il lato ovest del magazzino è caratterizzato da un muro perimetrale avente una lunghezza di 10,53 m (paramento esterno) e di 9,50 m (paramento interno). Quattro finestrelle orizzontali con angolo di lavorazione orientato verso il basso e verso l'interno, originariamente realizzate con due blocchi di pietra sovrapposti, dovevano portare luce e areazione al vano sottostante (fig. 17-18). 
Fig. 14 - Magazzino A. Prospetto del paramento interno del muro perimetrale nord.

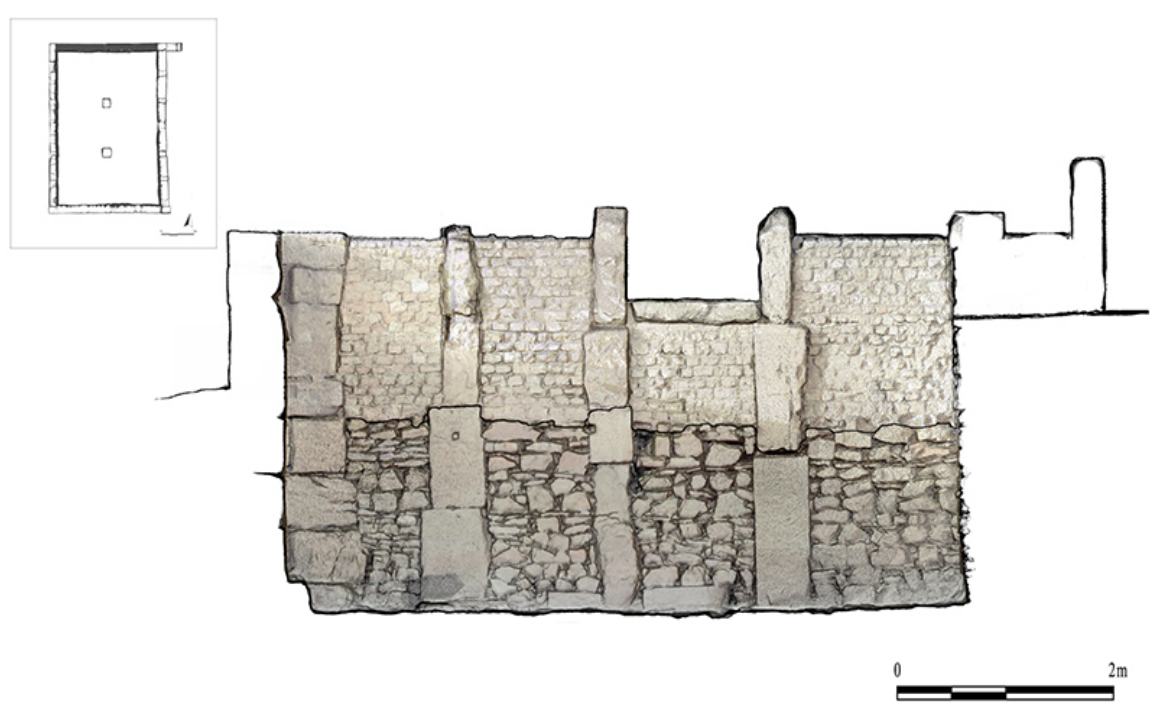

Fr. Martorella.

Fig. 15 - Magazzino A. Prospetto del paramento interno del muro perimetrale est.
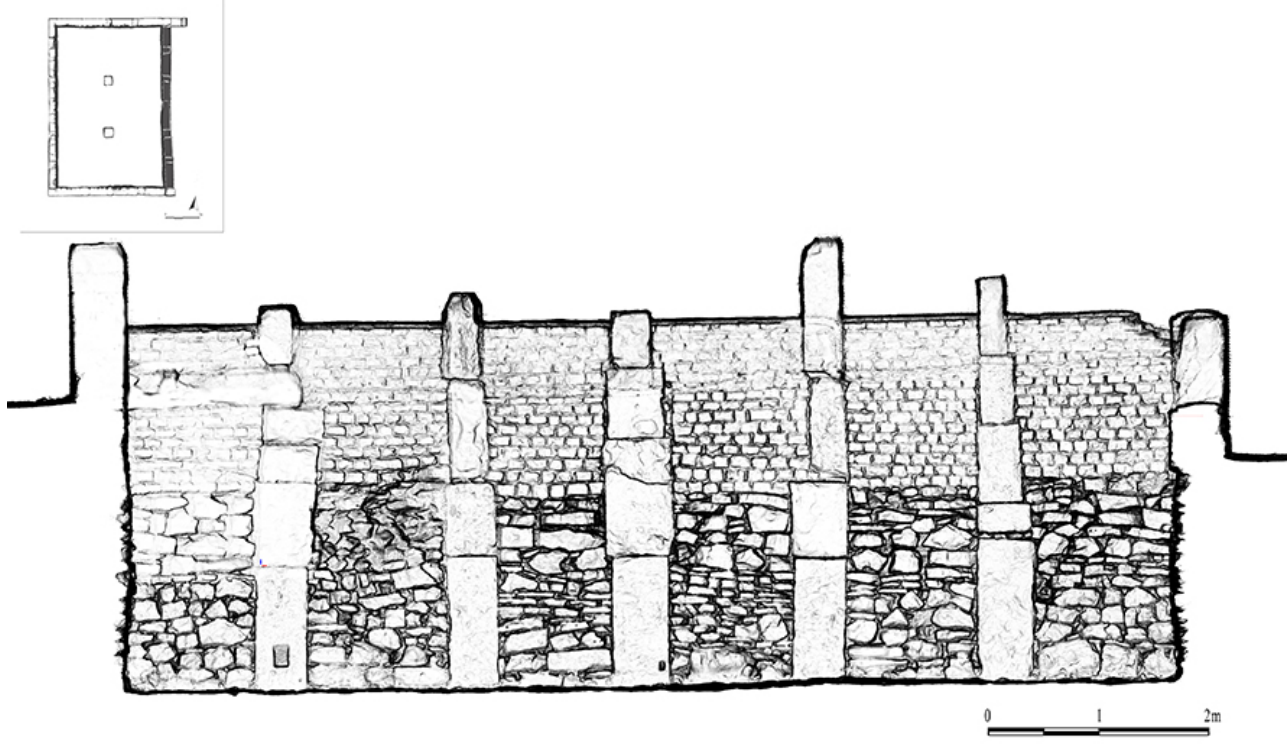

Fr. Martorella. 
Fig. 16 - Magazzino A. Prospetto del paramento interno del muro perimetrale sud con nuvole di punti sovrapposte al rilievo fotogrammetrico.

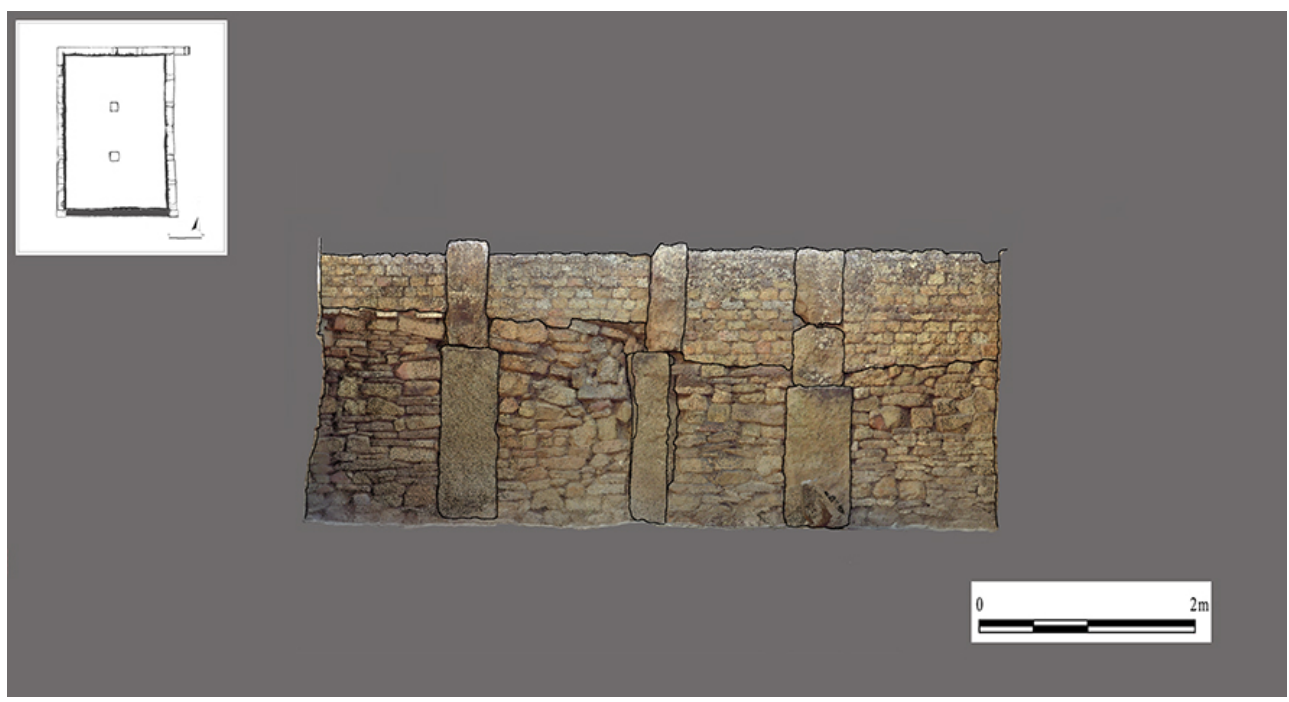

Fr. Martorella.

Fig. 17 - Magazzino A. Rilievo fotogrammetrico del muro perimetrale ovest con linea di demarcazione della parte superiore restaurata (rilievo fotogrammetrico).

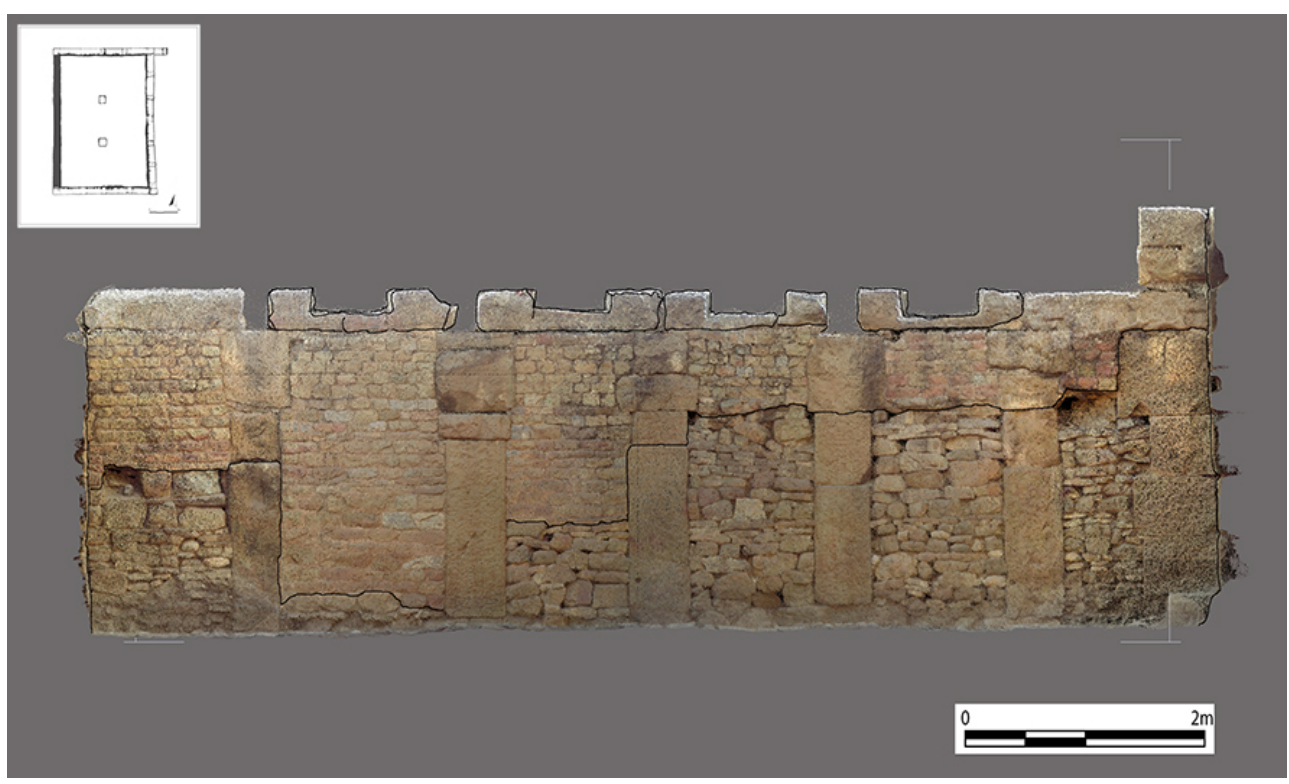

Fr. Martorella. 
Fig. 18 - Magazzino A. Muro perimetrale ovest. Particolare del blocco litico inferiore delle aperture (n. 1-4).

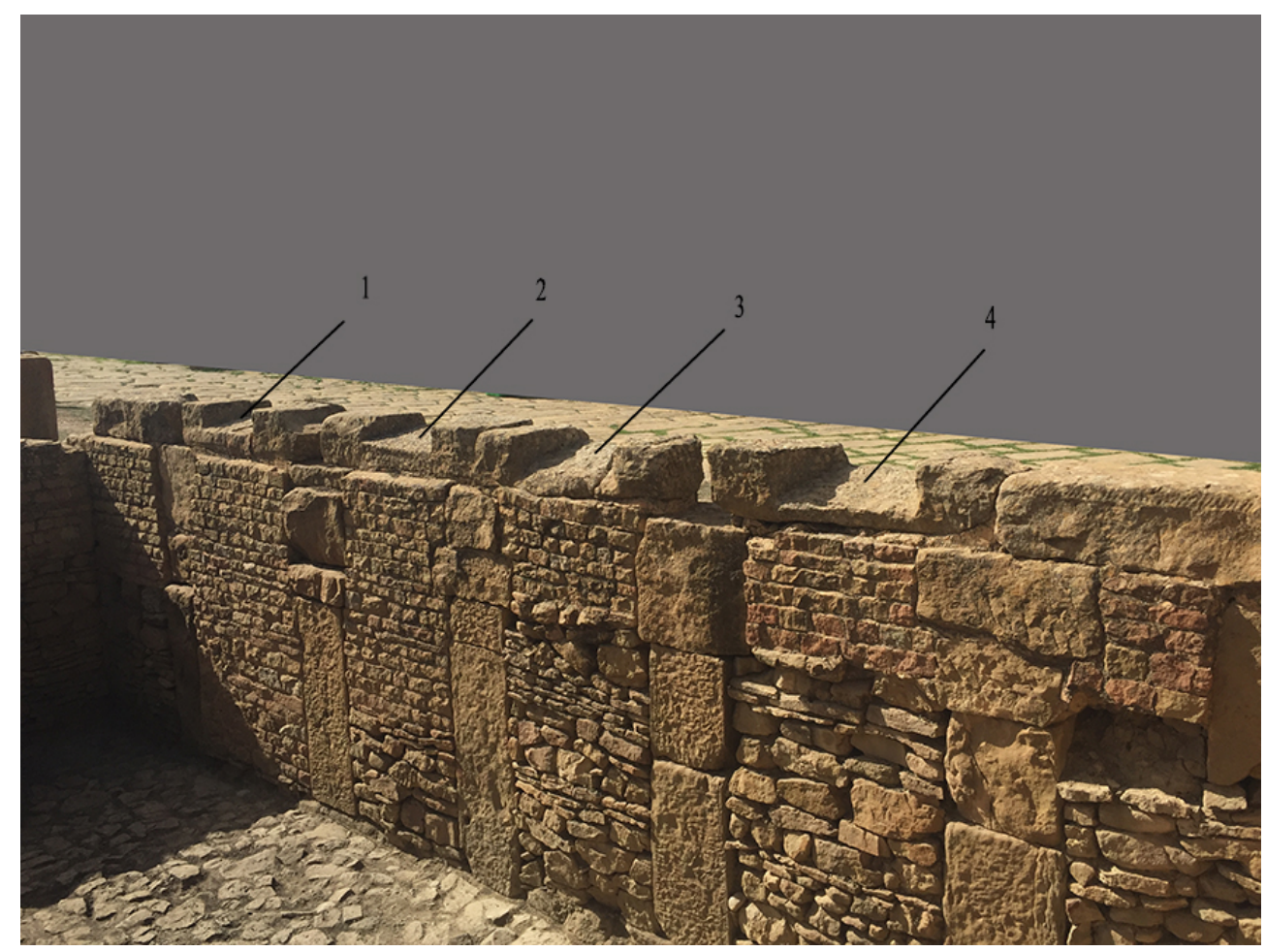

Fr. Martorella.

L'analisi architettonica dei muri perimetrali del magazzino A, seppur compromessa da una evidente azione di restauro nella parte superiore dei paramenti, ha permesso di comprendere la tecnica costruttiva utilizzata nell'allestimento degli alzati e l'organizzazione interna dell'edificio. Originariamente, si accedeva all'ambiente dal decumanus, attraverso una porta a doppio battente con apertura verso l'interno. Il piano pavimentale era costituito da un tavolato ligneo, non più conservato, supportato internamente da pilastri in pietra (non conservati) addossati ai muri perimetrali unitamente ai pilastri centrali ancora visibili. Attraverso una botola o una scala ricavata nel tavolato ligneo, si accedeva a un ambiente sottostante, areato e illuminato dalle aperture orizzontali previste lungo il muro perimetrale ovest. Il vano sottostante doveva raggiungere un'altezza di circa $3 \mathrm{~m}$. Il piano pavimentale del vano doveva essere costituito, in una fase di utilizzo dell'ambiente non meglio definita, da un piano di pietre di piccole e medie dimensioni.

\section{Magazzino B}

24 Il magazzino B si presenta come una struttura rettangolare di $87 \mathrm{~m}^{2}$, di cui $60 \mathrm{~m}^{2}$ calpestabili, realizzata come ambiente unico ma collegato con gli ambienti dell'insula (fig. 19-20). Lo spessore dei muri perimetrali nord, sud, est e ovest, realizzati anch'essi in opus africanum, è di $0,50 \mathrm{~m}$. L'edificio è stato interamente scavato dalle missioni francesi che hanno restaurato la parte sommitale dei paramenti dei muri perimetrali. 
Fig. 19 - Magazzino B. Elaborazione delle nuvole di punti con localizzazione della porta ovest, in trasparenza a sinistra, del decumanus con orientamento est-ovest e del magazzino B nell'angolo nord-ovest della terzùltima insula. (rilievo fotogrammetrico).



Fr. Martorella.

Fig. 20 - Particolare dell'insula e del magazzino B con relativi ingressi e accessi collegati.

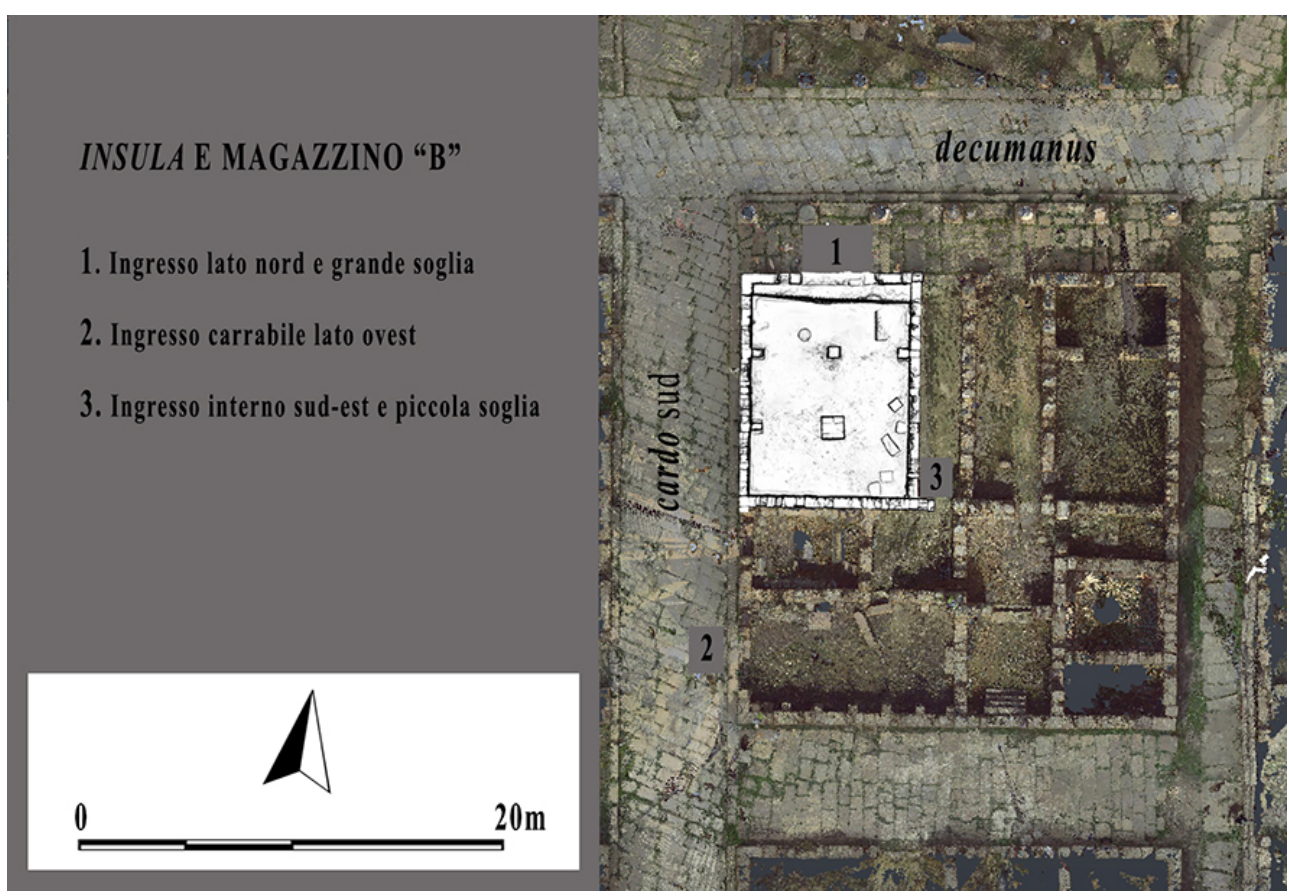

Fr. Martorella.

25 L'accesso al magazzino, anch'esso sul portico colonnato che fiancheggia il decumanus, si realizza attraverso una grande soglia d'ingresso di $2,80 \mathrm{~m}$ di lunghezza e $0,50 \mathrm{~m}$ di larghezza con apertura verso l'interno. Una piccola soglia nell'angolo sud-est collega il magazzino con il corridoio est (fig. 21). 
Fig. 21 - Pianta del magazzino B con indicazione della grande soglia nord e della piccola soglia sud-est (rosa).

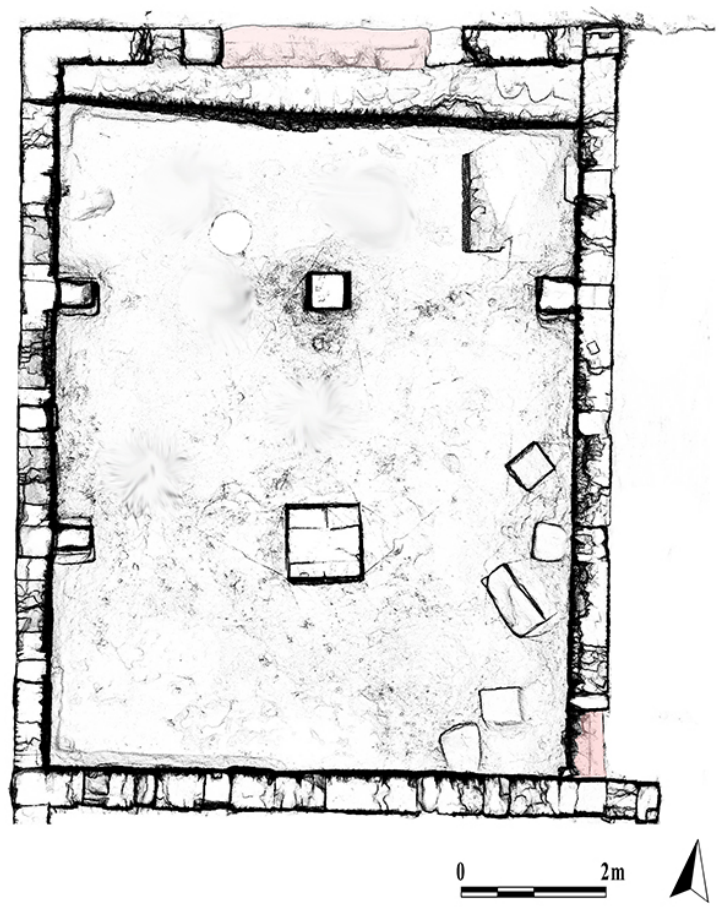

Fr. Martorella.

Diversamente dal magazzino A, in gran parte rimaneggiato, l'edificio B evidenzia, in posizione originaria, alcuni elementi costruttivi tipici dei magazzini con ambienti sopraelevati e soggetti a problematiche termo-igrometriche nocive alla conservazione delle derrate: pilastri interni e laterali a sostegno del tavolato ligneo non conservato; piano di calce isolante e strato di cocciopesto con la medesima funzione steso sui paramenti interni; muri perimetrali con finestre di illuminazione e areazione del vano sottostante previste nel muro perimetrale ovest.

\section{Elementi sostruttivi: i pilastri e il muro basso nord}

Il tavolato ligneo, non conservato e in quota con le soglie nord e sud-est, copriva l'ambiente nella sua interezza; era sostenuto da pilastri in grossi blocchi di pietra posti ai lati est e ovest dell'ambiente e appoggiati ai muri perimetrali. In posizione centrale, in asse con i pilastri laterali, due pilastri in blocchi di pietra dovevano avere la funzione di rompitratta a sostegno dell'impiantito ligneo. Il pilastro centrale nord presenta una larghezza di $0,50 \mathrm{~m}$ per lato. Il pilastro centrale sud presenta, invece, dimensioni più imponenti $(1 \times 1,20 \mathrm{~m})$, verosimilmente dovute a un suo maggiore ruolo sostruttivo; è plausibile che il tavolato ligneo doveva essere sottoposto a una maggiore sollecitazione nella parte sud in considerazione di un secondo accesso nell'angolo sud-est dove è ancora visibile una piccola soglia di $1 \mathrm{~m}$ di lunghezza e 0,48 $\mathrm{m}$ di larghezza. Due blocchi di pietra ancora in situ e in fase originaria nell'angolo sud-est avevano la funzione di sostenere alla base una scala di collegamento con il vano sotto il tavolato a cui si accedeva attraverso una botola prevista nel tabulatum (fig. 22). 
Fig. 22 - Magazzino B, con indicazione dei pilastri (verde) e del muro basso nord (giallo e arancione) a sostegno del tavolato ligneo; basi in pietra (azzurro) a sostegno di una scala di collegamento con il vano sottostante.

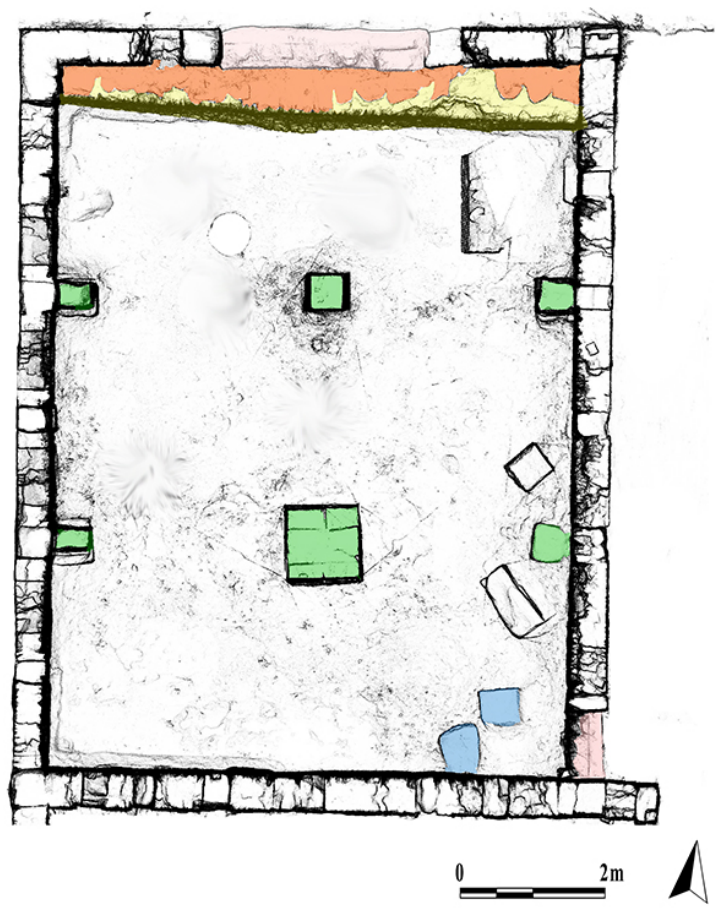

Fr. Martorella.

28 A nord del vano, in corrispondenza della grande soglia, il tavolato ligneo doveva essere sostenuto da un muro basso (altezza conservata di $1,45 \mathrm{~m}$ ), realizzato in pietre di piccole e medie dimensioni disposte per piano e completato con un secondo livello di pietre anch'esse disposte per piano. Il muro basso con andamento est-ovest presenta una lunghezza di $7,25 \mathrm{~m}$ e uno spessore di $0,78-0,50 \mathrm{~m}$. Appoggiandosi ai muri perimetrali est e ovest, doveva costituire la base nord di appoggio al tavolato ligneo in un punto sottoposto a forti sollecitazioni (ingresso).

Le indagini archeologiche potranno chiarire se la realizzazione del muro basso è in fase con il completamento dell'edificio oppure se il suo allestimento si è reso necessario in un momento successivo di riorganizzazione degli spazi per offrire maggiore sostegno al muro perimetrale nord e un più consistente supporto al tavolato ligneo (fig. 23). Il muro basso si appoggia al muro perimetrale nord (lungh. esterna $8,45 \mathrm{~m}$ ) che si presenta quasi totalmente restaurato (fig. 24). 
Fig. 23 - Magazzino B. Prospetto interno del muro basso nord con orientamento est-ovest. In evidenza il muro basso (giallo) e l'ultimo livello di pietre anch'esse disposte per piano (arancione).
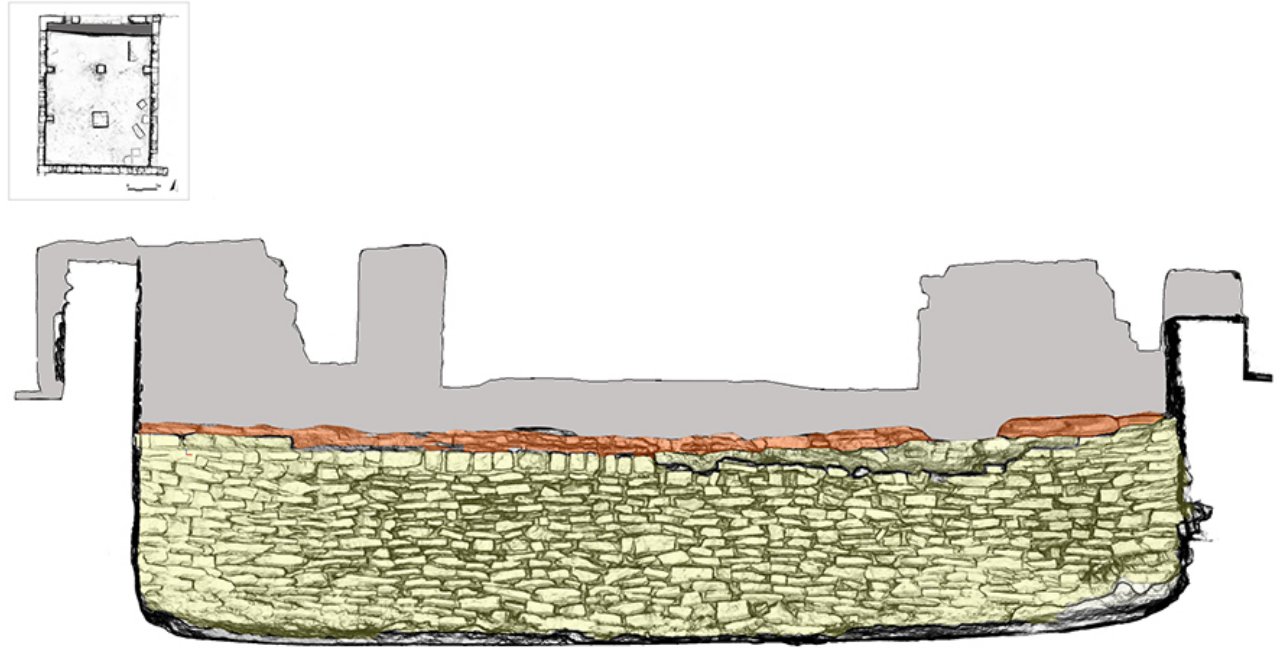

0 $2 \mathrm{~m}$

Fr. Martorella.

Fig. 24 - Magazzino B. Prospetto interno del muro perimetrale nord. In evidenza la grande soglia di ingresso (rosa).
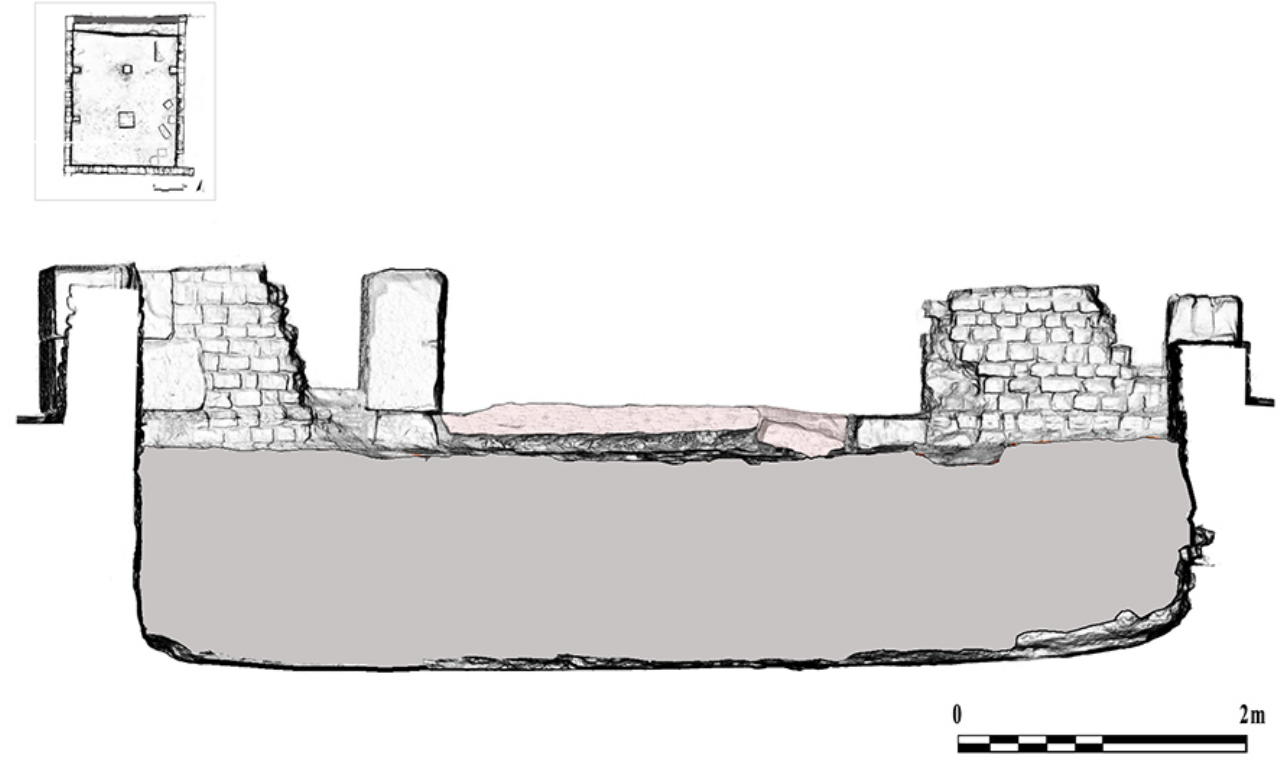

Fr. Martorella.

\section{Piano di calce isolante e strato di cocciopesto}

30 L'interno del vano doveva essere realizzato in modo estremamente accurato. La pulizia dell'ambiente e l'analisi delle strutture interne e dei paramenti hanno evidenziato uno strato di calce compatto, ben conservato e con pendenza verso nord, steso sul piano pavimentale. Nei punti di contatto con i muri perimetrali e negli angoli nord-ovest, 
nord-est e sud-ovest dell'ambiente è conservato uno strato molto compatto di cocciopesto (fig. 25).

Fig. 25 - Piano di calce del vano sottostante (ocra) con indicazione dello strato di cocciopesto negli angoli sud-ovest, nord-ovest, nord-est e lungo la parete interna ovest (ocra scuro).

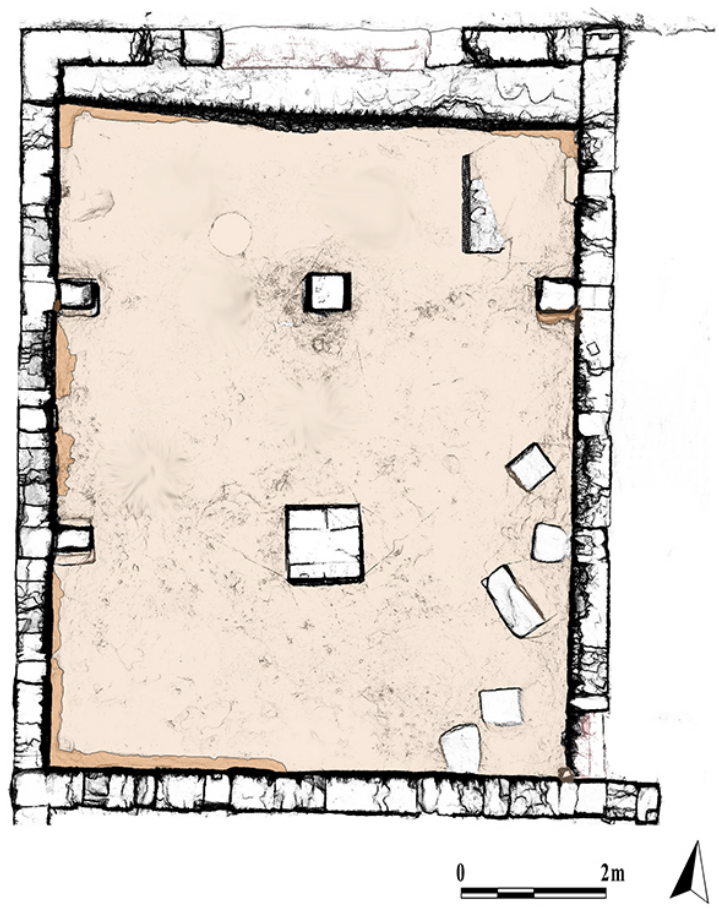

Fr. Martorella.

31 Uno strato isolante di calce con pendenza verso nord doveva rivestire l'interno di tutto l'ambiente. Il paramento ovest, evidentemente soggetto a problematiche termoigrometriche assai nocive, conserva ancora lembi di uno strato di cocciopesto nei punti di collegamento con il piano pavimentale di calce e in corrispondenza dei pilastri interni (fig. 26-28). 
Fig. 26 - Strato di calce isolante sul piano pavimentale e strato di cocciopesto (contorno nero) con funzione isolante sul paramento interno del muro ovest (particolare).

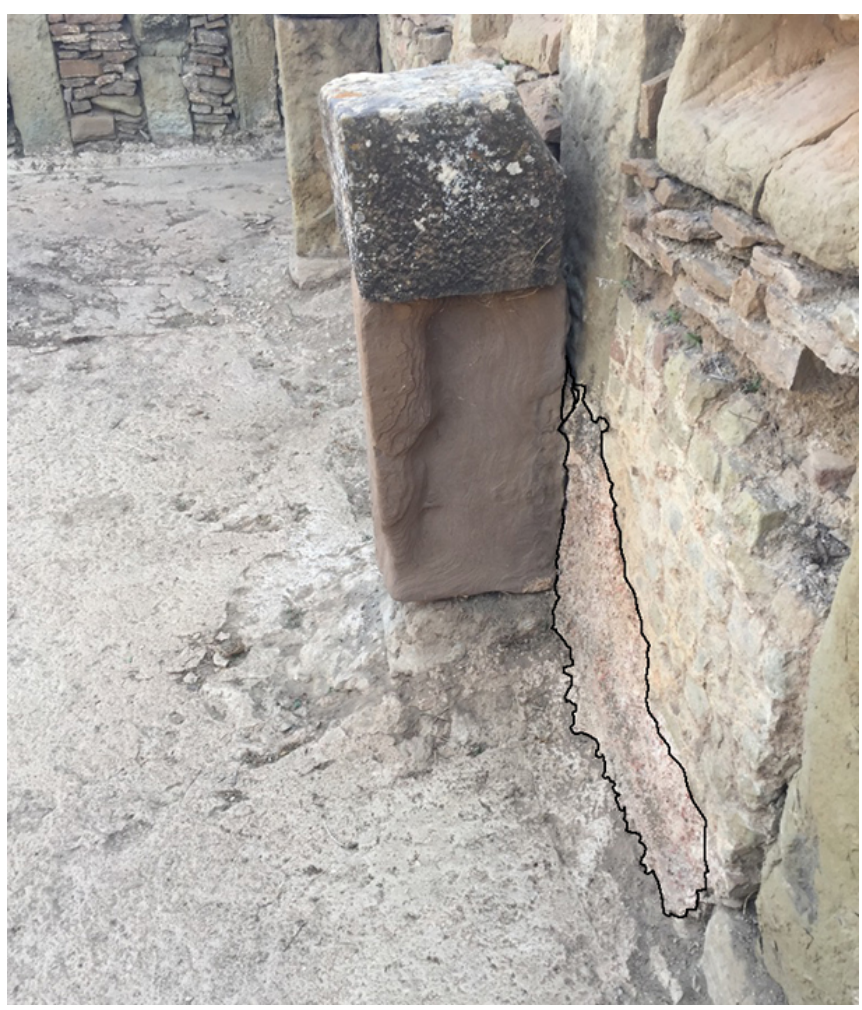

Fr. Martorella.

Fig. 27 - Magazzino B. Prospetto interno del muro perimetrale ovest. Tracce di cocciopesto (ocra scuro).
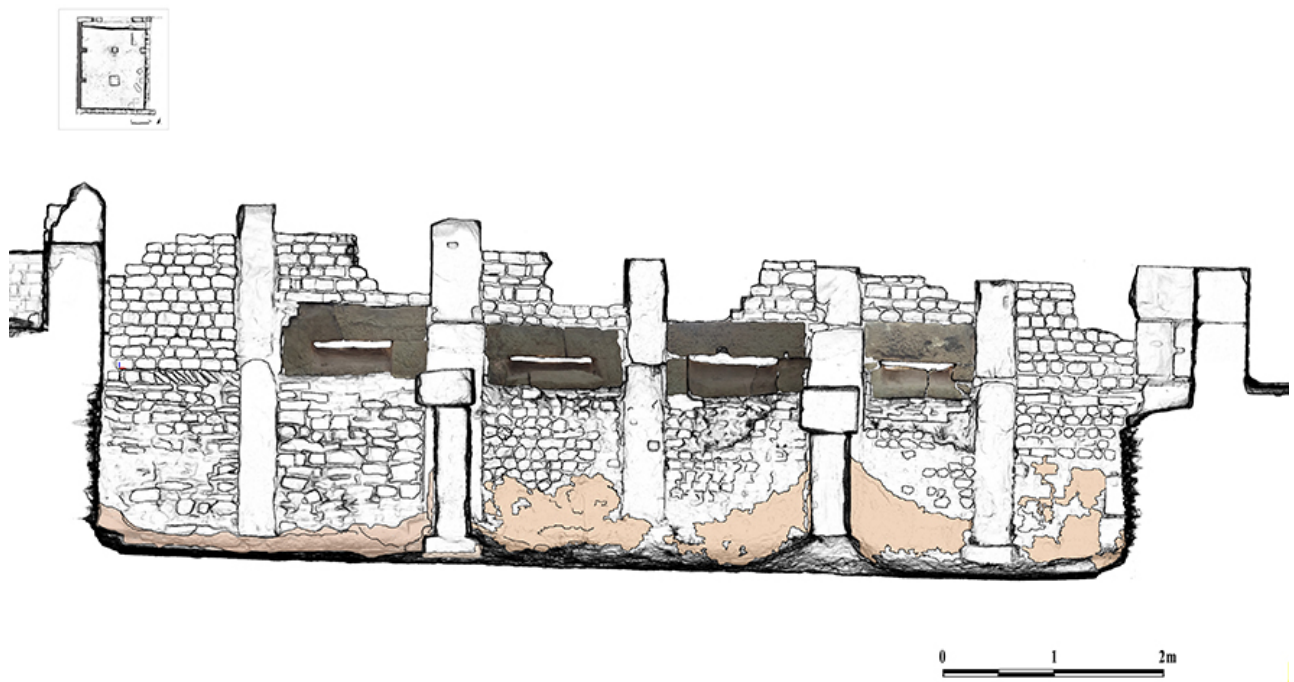

Fr. Martorella. 
Fig. 28 - Strato di rivestimento di cocciopesto molto compatto nell'angolo sud-ovest e sul piano pavimentale.

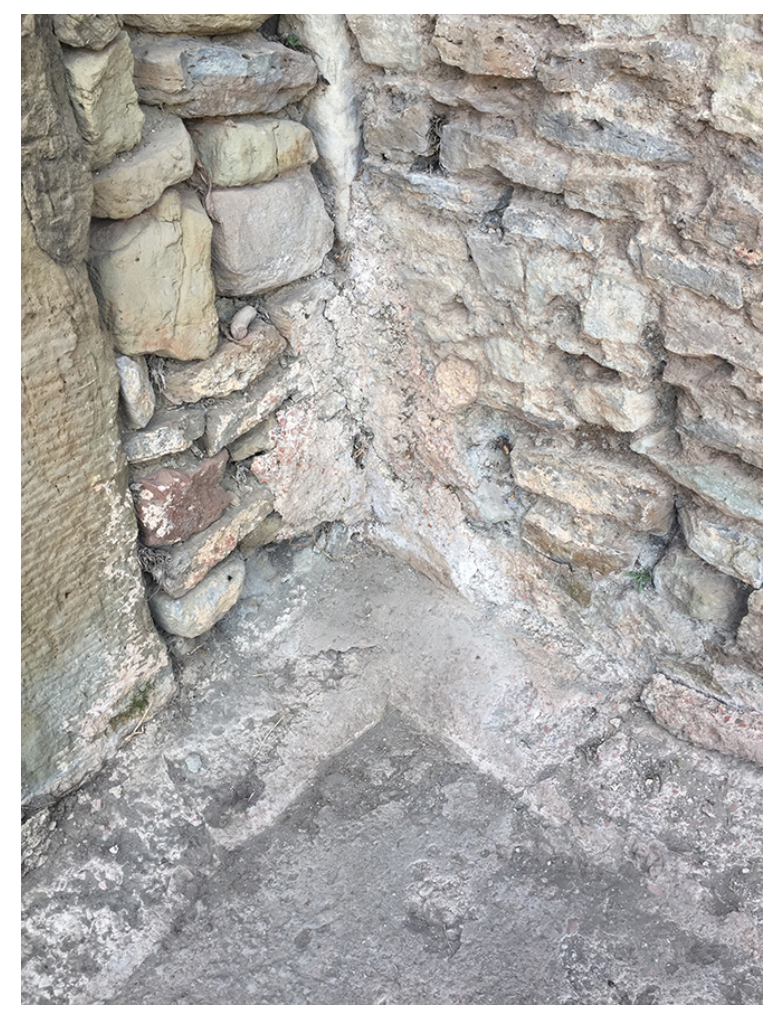

Fr. Martorella.

\section{Muri perimetrali e finestrelle di illuminazione e areazione}

Il magazzino era delimitato da muri perimetrali ben allestiti in opus africanum con pilastri di blocchi di lunghezza variabile sovrapposti in verticale. Si tratta di blocchi in pietra lavorati a squadro e indipendenti rispetto ai paramenti intermedi apparecchiati con pietre di differenti dimensioni disposte in maniera irregolare (parte inferiore dei paramenti) e pietre a bozzetti disposte in modo più o meno regolare (parte superiore dei paramenti $)^{10}$. In età imperiale, questo tipo a telaio con pilastri sovrapposti risulta il più diffuso e utilizzato nella costruzione di altri importanti insediamenti (Tiddis, Cuicul). L'uso dei pilastri in blocchi di pietra doveva garantire la ripartizione dei carichi; le terminazioni litiche trasferivano il peso della costruzione direttamente sulle fondazioni dell'edificio. Il muro perimetrale est presenta una lunghezza di 9,60 m (paramento esterno) e di $8,20 \mathrm{~m}$ (paramento interno) e delimitava l'ambiente con il corridoio orientale a cui si accedeva attraverso il piccolo ingresso posto nell'angolo sud-est (fig. 29). 
Fig. 29 - Magazzino B. Prospetto del paramento interno del muro est. A destra, piccola soglia (rosa) di accesso al corridoio.
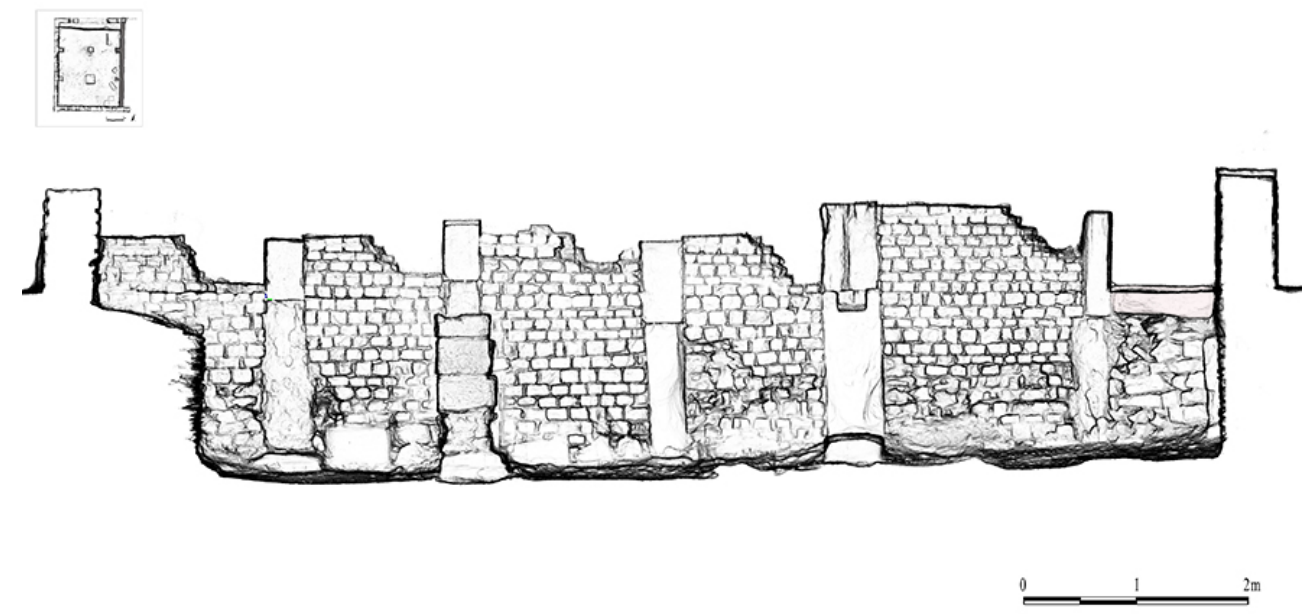

Fr. Martorella.

La parte meridionale è delimitata dal muro sud con una lunghezza di $8,20 \mathrm{~m}$ (paramento esterno) e di $7 \mathrm{~m}$ (paramento interno) (fig. 30). La lunghezza del muro perimetrale ovest (paramento esterno) è di $10,70 \mathrm{~m}$. Come per il magazzino $\mathrm{A}$, anche il magazzino B presenta sul muro perimetrale ovest delle finestrelle orizzontali con angolo di lavorazione inclinato verso il basso (fig. 31-32). Le aperture $(0,70 \times 0,15 \mathrm{~m} \mathrm{ca})$ sono contornate da due blocchi litici sovrapposti. Lo spessore dei blocchi è pari a quello dei muri perimetrali $(0,50 \mathrm{~m})$. Come il blocco inferiore ( $\mathrm{vd}$. anche aperture del magazzino $\mathrm{A}$, fig. 18), così il blocco superiore delle aperture presenta un'inclinazione verso il basso e medesima lavorazione (fig. 33). A differenza del magazzino A, il magazzino $B$ doveva caratterizzarsi per una più limitata agibilità interna se si considera l'altezza tra il piano di calce e il tavolato $(1,80 \mathrm{mca})$. Le finestrelle orizzontali dovevano risultare, dunque, una soluzione molto efficace per areare il vano sotto il tavolato, creare un vuoto sanitario e soddisfare le esigenze termo-igrometriche dell'intercapedine (fig. 34). 
Fig. 30 - Magazzino B. Prospetto del paramento interno del muro sud. A dx strato di cocciopesto (ocra scuro).

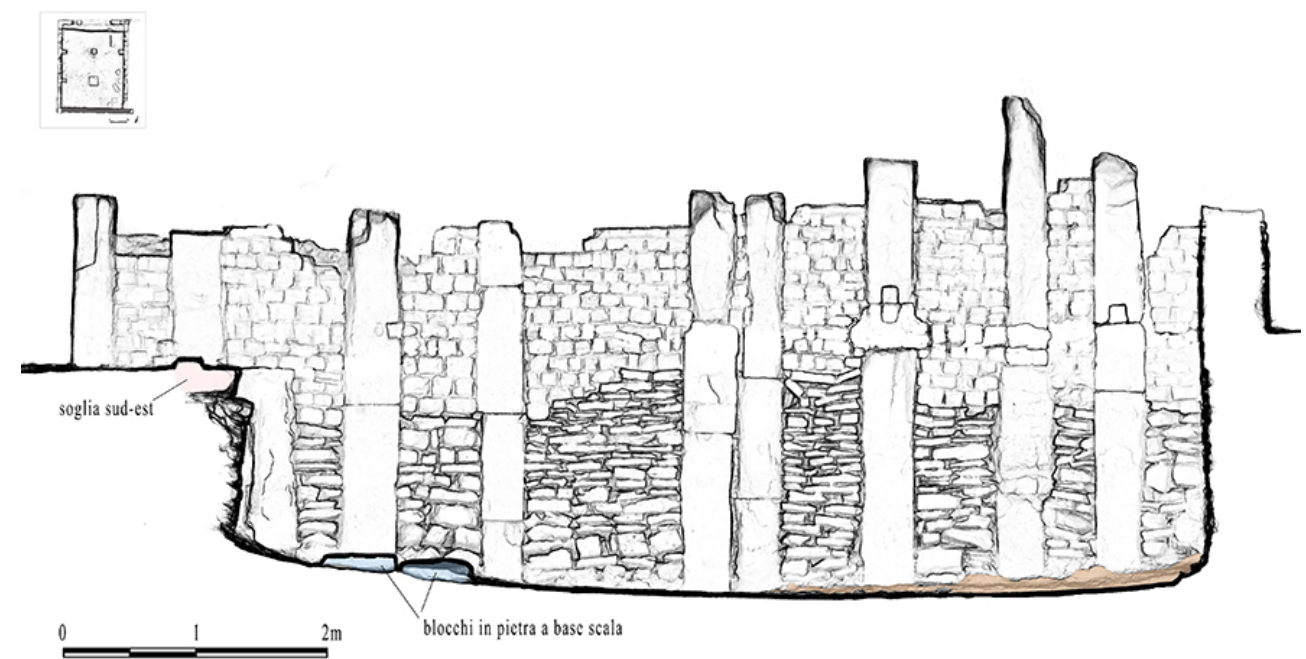

Evidente la linea di demarcazione tra differenti apparecchiature compromesse, inoltre, da una forte azione di restauro nella parte superiore dell'alzato.

Fr. Martorella.

Fig. 31 - Magazzino B. Prospetto interno muro perimetrale ovest con orientamento nord-sud.

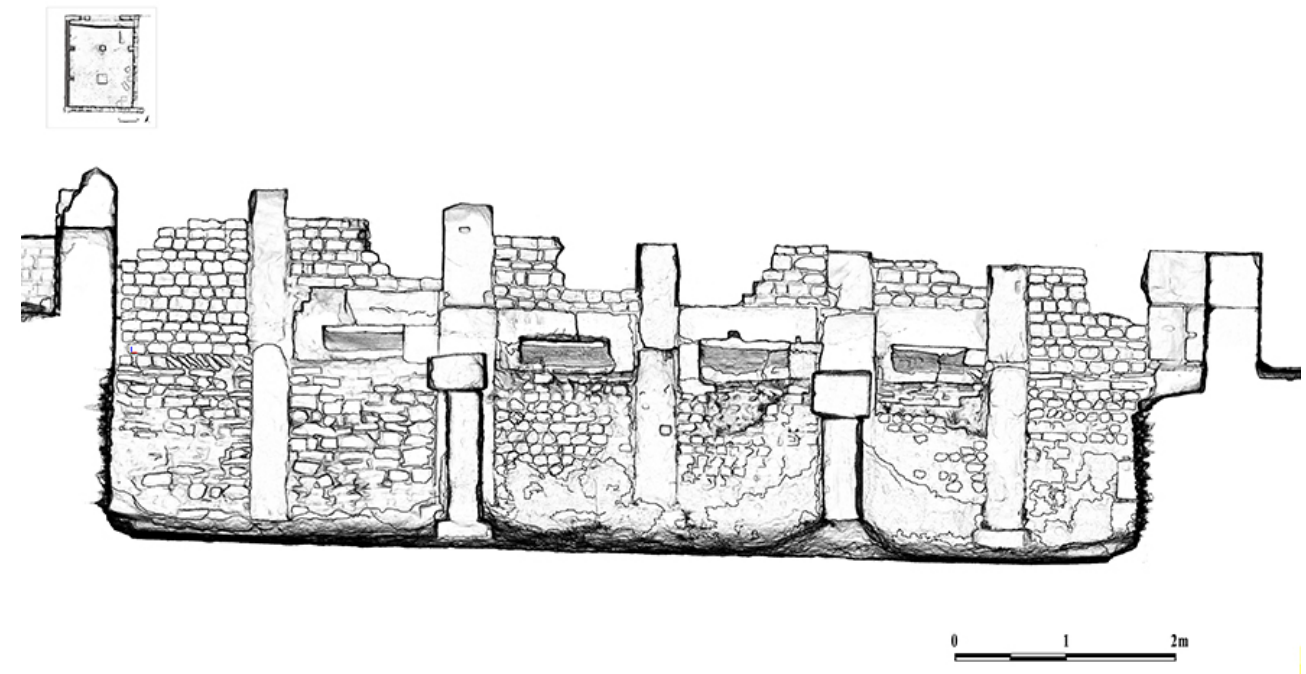

Fr. Martorella. 
Fig. 32 - Magazzino B. Prospetto interno muro ovest con le aperture orizzontali in evidenza (rilievo fotogrammetrico).

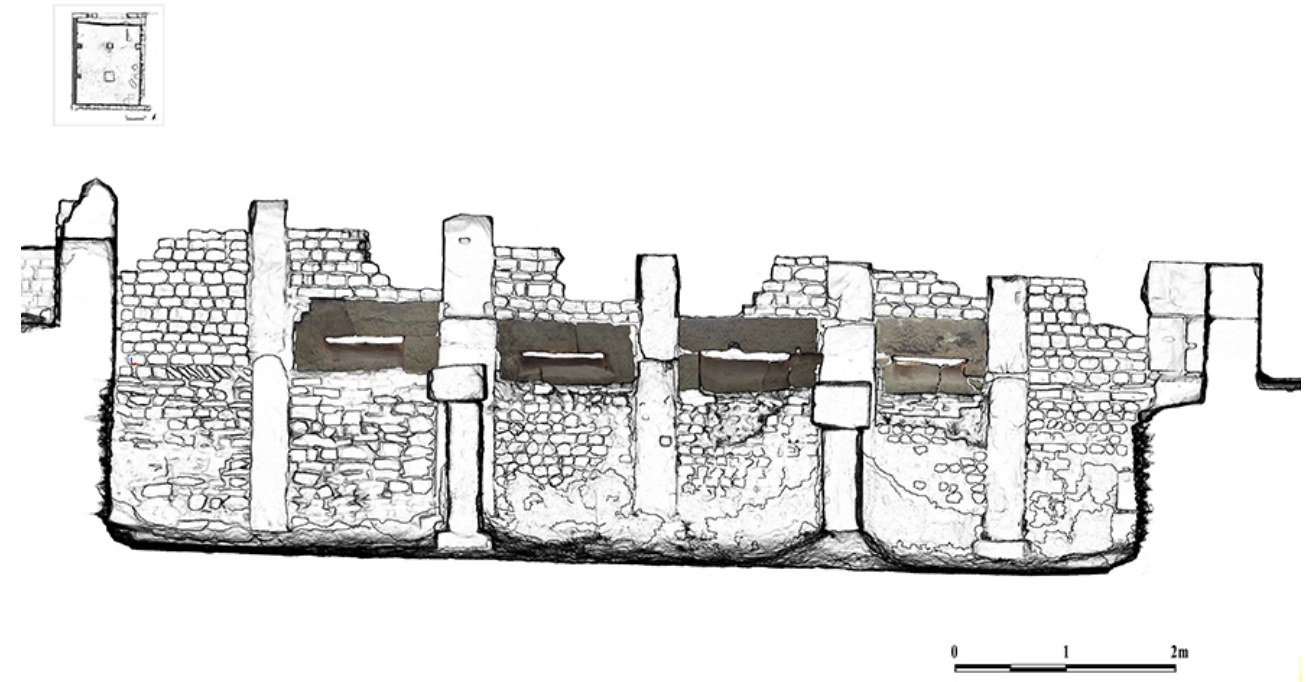

Fr. Martorella.

Fig. 33 - Magazzino B. Muro perimetrale ovest (areatore 2) con angolo di inclinazione del blocco litico superiore (part.).



Fr. Martorella. 
Fig. 34 -Magazzino B. Sezione A-A' e livelli di quota (muro insula ovest; marciapiede ovest; cardo; marciapiede est; areatore; livello calce isolante; soglia e livello del tavolato ligneo).

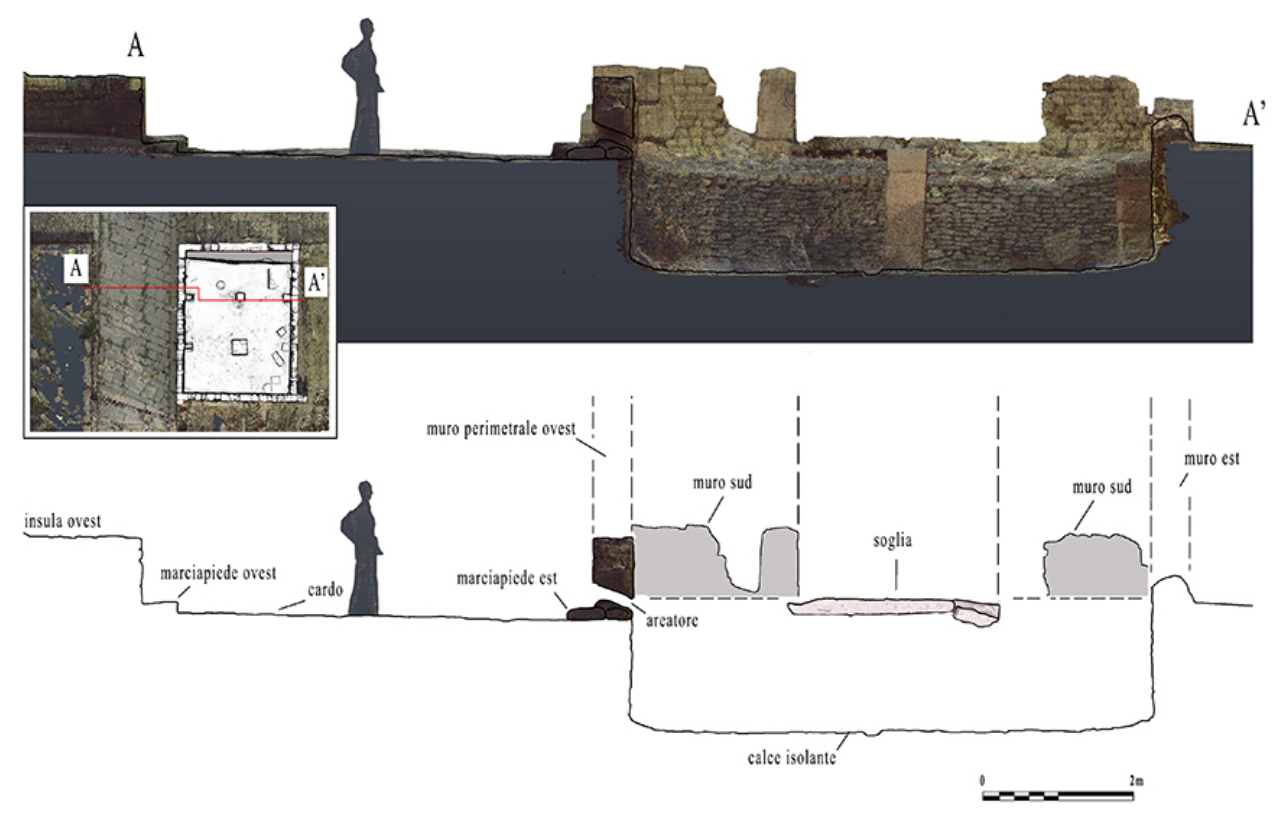

Fr. Martorella.

34 All'esterno, blocchi di pietra rettangolari alti $0,20 \mathrm{~m} c a$ ai lati del cardo proteggevano le aperture del magazzino da possibili infiltrazioni di acqua piovana. Uno strato di cocciopesto molto compatto rinvenuto tra i blocchi in pietra laterali e il muro perimetrale ovest doveva, verosimilmente avere la stessa funzione isolante (fig. 35). 
Fig. 35 - Magazzino B. Areatore 1 (nord) e strato di cocciopesto (part.) tra il muro perimetrale ovest e il limite orientale del cardo.

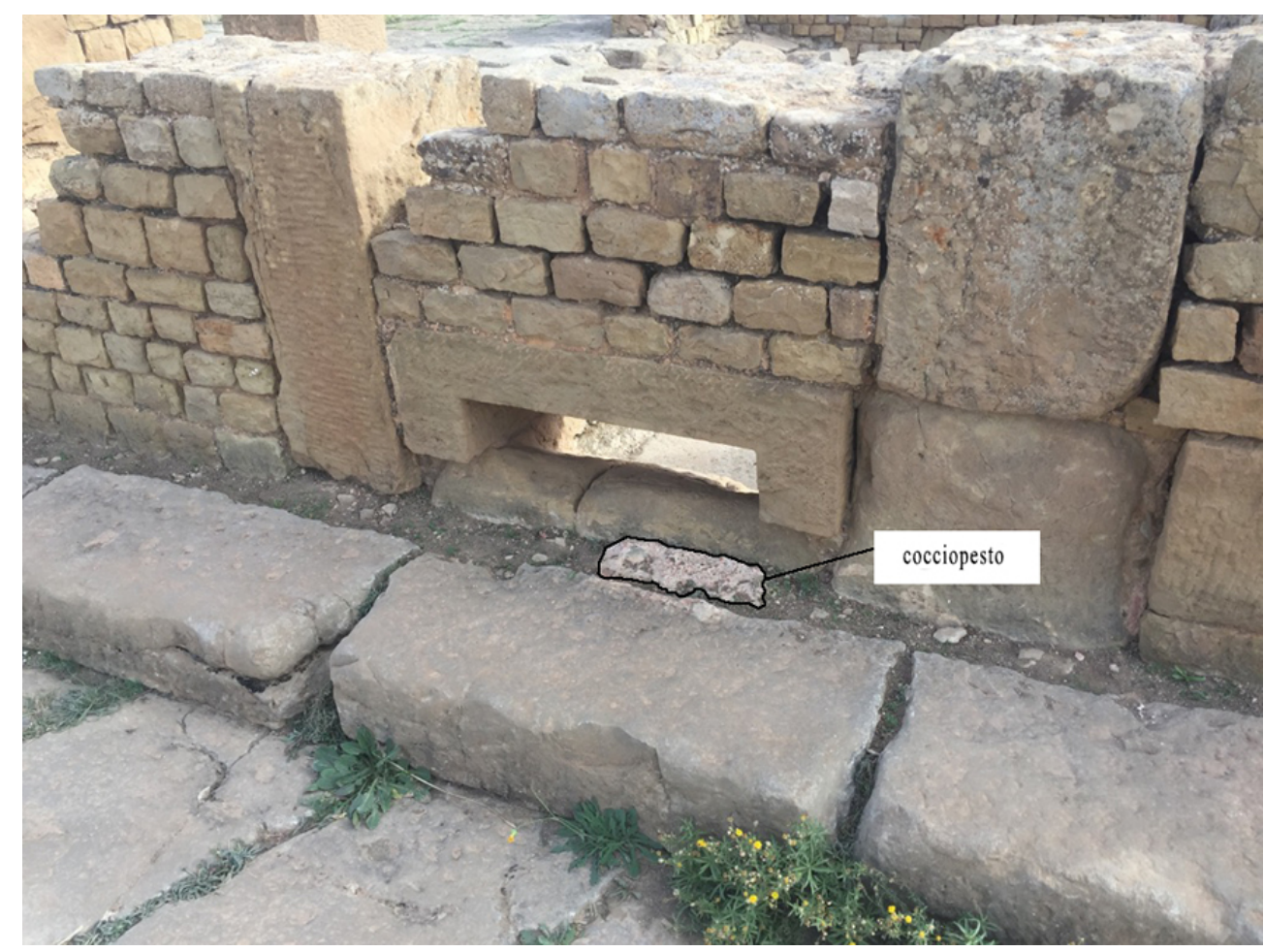

Fr. Martorella.

\section{Conclusioni dell'analisi strutturale}

La molteplicità dei dati ottenuti consente di considerare e identificare entrambi gli edifici come magazzini cittadini con caratteristiche peculiari di accessibilità, salubrità e sicurezza statica ${ }^{11}$.

Il magazzino A è collocato al limite ovest della città, in un'area soggetta a una evidente espansione urbanistica fuori del reticolato primitivo. Non collegato con altri ambienti retrostanti e laterali, il magazzino si presenta come un edificio indipendente con un unico accesso a nord dal decumanus. Non si conservano i pilastri interni a sostegno del tavolato ligneo che non doveva essere inferiore alla soglia di ingresso. Si conservano due tramezzi centrali in forma di pilastri di pietra e un livello pavimentale costituito da pietre di piccole e medie dimensioni relativo al vano seminterrato.

Il dato caratteristico e comune all'edificio $B$ è rappresentato da quattro aperture di areazione/ventilazione/illuminazione previste sul muro ovest. Di queste si conservano i blocchi di pietra inferiori con un caratteristico angolo di lavorazione verso il basso (vd. fig. 15 e 16). Non sono state rinvenute tracce di rivestimenti parietali interni ed esterni.

L'edificio B, anch'esso con accesso dal decumanus, è localizzato nell'angolo nord-ovest della terz'ultima insula e sembra essere parte integrante dell'abitazione che lo fiancheggia a sud e a est. Nell'analisi dei percorsi, le attività connesse con il magazzino B erano rese agevoli da un secondo grande ingresso di collegamento diretto con il cardo, sul lato sud-ovest dell'edificio (fig. 36, n. 2). Al vano sud-ovest si poteva 
accedere con un piccolo carro come testimoniano le tracce evidenti di usura sulla grande soglia con apertura a doppio battente (fig. 37).

Fig. 36 - Particolare dell'insula e del magazzino B con relativi ingressi collegati.

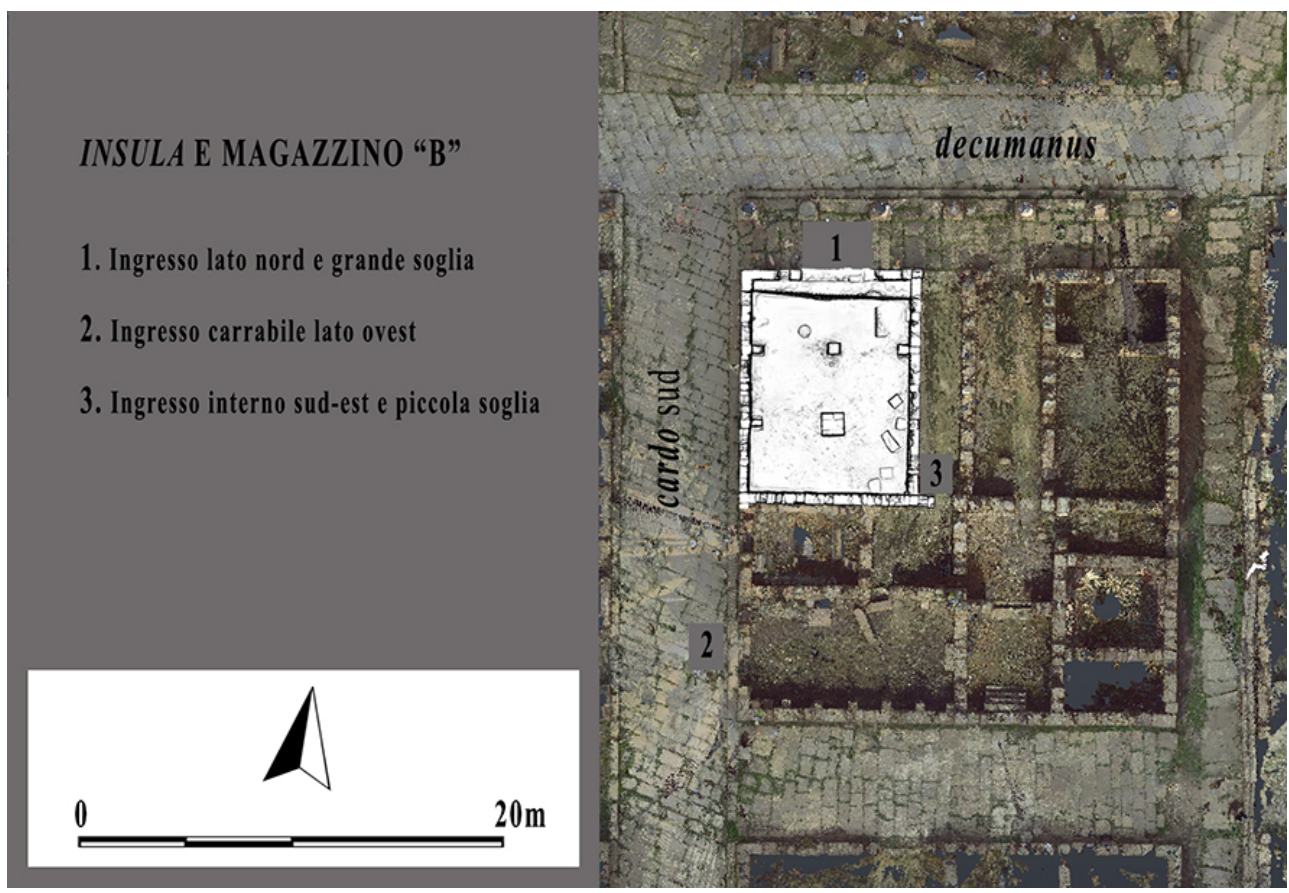

Fr. Martorella.

Fig. 37 - Soglia carrabile lungo il cardo (vd. fig. 34, n. 2) del vano sud-ovest della terz'ultima insula.



Fr. Martorella. 
elementi strutturali presenti nel magazzino B consentono una più agevole ricostruzione dell'edificio. Le attività relative alla sua organizzazione interna dovevano svolgersi, dunque, utilizzando l'ingresso laterale posto lungo il cardo. L'accesso principale al magazzino avveniva attraverso la grande soglia a nord. Un tavolato ligneo ben allestito costituiva il piano di utilizzo dell'ambiente.

Il piano in legno era sostenuto da pilastri in grossi blocchi di pietra. Il sistema doveva essere quello della contabulatio a travature incrociate, sostenuta da un impianto ad appoggio semplice su blocchi in pietra. Al centro del magazzino, i blocchi di pietra, ancora in situ, squadrati e con funzione di tramezzi, assicuravano un ulteriore punto d'appoggio all'intera orditura. I blocchi accoglievano nel senso della lunghezza travi posizionate secondo il sistema a due appoggi semplici (fig. 38).

Fig. 38 - Magazzino B. Rilievo fotogrammetrico e assonometrico con ipotesi ricostruttiva del tabulatum.



Fr. Martorella.

I locali seminterrati sono sempre soggetti a infiltrazioni di umidità e ad altri problemi dovuti alla scarsa aerazione. Oltre a questo, la scarsa luminosità naturale dei locali rende in genere molto bassa la loro vivibilità.

Come per il magazzino A, anche nel magazzino B, lungo i muri perimetrali occidentali erano previste quattro aperture orizzontali di areazione, realizzate con blocchi litici sovrapposti e angolo di lavorazione inclinato verso il basso; la particolare forma dei blocchi aveva l'obiettivo di fornire luce al vano sottostante, ma, soprattutto, di favorire la ventilazione dell'ambiente, mantenendo condizioni di temperatura e umidità adatte alla conservazione delle derrate alimentari. Il sistema consentiva l'ingresso da ovest di correnti di aria sotto il tavolato ligneo. Probabilmente, grate in legno o in ferro previste ai lati delle aperture impedivano l'ingresso di roditori o altri animali nocivi ${ }^{12}$.

Nel caso del magazzino A, il vano sotto il tavolato doveva presentarsi come un ambiente agibile se si considera l'altezza superiore ai 3 metri e accessibile, come per il magazzino B, attraverso una botola prevista nel tabulatum. In entrambi i magazzini, le indagini hanno constatato l'assenza di altri areatori previsti nei muri perimetrali est, 
nord e sud. L'areazione e la ventilazione dei vani doveva essere garantita solo dall'esterno, vale a dire dal lato ovest fiancheggiato dalle rispettive strade.

Solo il magazzino $\mathrm{B}$ ha evidenziato uno strato di calce isolante sul piano pavimentale e tracce di cocciopesto molto compatto. Tali rivestimenti, tipici dei magazzini destinati alla conservazione delle granaglie, sono stati rinvenuti negli angoli, sui paramenti interni e nei punti di giunzione tra i muri perimetrali e il piano pavimentale di calce. Lembi di cocciopesto con la medesima funzione isolante sono stati rinvenuti anche all'esterno dell'edificio, tra il marciapiede ovest e il muro perimetrale ovest. Si tratta anche in questo caso di azioni finalizzate a proteggere il magazzino dal rischio di infiltrazioni di acqua piovana e dal pericolo di annidamento di insetti o animali nocivi.

Purtroppo, gli scavi precedenti e le attività di restauro hanno cancellato tracce archeologiche importanti relative alla cronologia degli edifici. Tuttavia, il prosieguo delle ricerche e l'avvio di indagini archeologiche potranno chiarire aspetti importanti della vita dei due magazzini, aggiungendo un tassello importante alla economia urbana della città di Thamugadi.

Fr. Martorella.

\section{BIBLIOGRAFIA}

Ballu 1897 = A. Ballu, Les ruines de Timgad, antique Thamugadi, Paris, 1897.

Ballu 1902 = A. Ballu, Théâtre et forum de Timgad (antique Thamugadi), état actuel et restauration, Ernest Leroux, Paris, 1902.

Boeswillwald - Cagnat - Ballu $1905=$ E. Boeswillwald, R. Cagnat, A. Ballu, Timgad une cité africaine sous l'Empire romain, Paris, 1905.

Camporeale 2013 = S. Camporeale, Opus Africanum e tecniche a telaio litico in Etruria e Campania (VII a.C.-VI d.C.), dans Archeologia dell'architettura, 18, 2013, p. 192-209.

Chankowski - Lafon - Virlouvet 2018 = V. Chankowski, X. Lafon, C. Virlouvet (dir.), Entrepôts et circuits de distribution en Méditerranée antique, Paris, 2018.

Christofle $1935=$ M. Christofle, Rapports sur les travaux de fouilles et de consolidation exécutés en 1930-1931-1932 à Timgad par les services des monuments historiques, dans Journal Officiel, 1935.

Courtois $1951=$ C. Courtois, Timgad : antique Thamugadi, Alger, 1951 .

Gentry 1976 = A.P. Gentry, Roman military stone-built granaries in Britain, Oxford, 1976 (BAR International Series, 32).

Giuliani 2006 = C.F. Giuliani, L'edilizia nell'antichità, Roma, 2006.

Groslambert 1997 = A. Groslambert, L'archéologie algérienne de 1895 à 1915, Les rapports d'Albert Ballu publiés au journal officiel de la République Française de 1896 à 1916, Lyon, 1997 (Collection du Centre d'études romaines et gallo-romaines, n. s., 16).

Papi - Martorella 2007 = E. Papi, F. Martorella, I granai della Numidia, dans Antiquités africaines, 43, 2007, p. 171-186. 
Thébert 2003=Y. Thébert, Thermes romains d'Afrique du Nord et leur contexte méditerranéen, études d'histoire et d'archéologie, Rome, 2003.

Virlouvet 2007 = C. Virlouvet, Entrepôts de stockage, entrepôts et marchés. Pour une typologie des horrea dans l'Afrique du Nord antique, dans Antiquités africaines, 43, 2007, p. 165-169.

\section{NOTE}

1. https://www.m-culture.gov.dz/index.php/fr/.

2. http://ogebc.com/site/fr/thamugadi-timgad.

3. Christofle 1935, p. 81.

4. Groslambert 1997, p. 13.

5. Boeswillwald - Cagnat - Ballu 1905, p. 334.

6. Id., p. 335.

7. Thébert 2003, p. 247.

8. Nous remercions le ministère de la culture algérien, l'office national de la gestion et d'exploitation des biens culturels protégés de nous avoir permis de faire le relevé des deux entrepôts de Timgad et l'École française pour les deux séjours à Rome.

9. https://www.faro.com/en-gb/products/construction-bim-cim/faro-focus/.

10. Sulla tipologia delle tecniche a telaio litico con pilastri nel Mediterraneo occidentale Camporeale 2013, p. 195-198. Sulla funzione statica della tecnica a telaio litico Giuliani 2006, p. 27-32, 142-143, 145-146.

11. Sull'identificazione dei due edifici come magazzini e più in generale sui granai della Numidia Papi - Martorella 2007, p. 172. Sulla tipologia dei magazzini e degli horrea nell'Africa del nord Virlouvet 2007, p.165-169. Sulle problematiche dell'immagazzinamento, dei modi di distribuzione e della speculazione nei circuiti economici del mondo greco e romano Chankowski - Lafon - Virlouvet 2018.

12. Gentry 1976, p. 11. Anche nei magazzini di I sistemi adottati nella cura dei locali e delle derrate stoccate non pone distinzioni tra magazzini statali o privati, cittadini o rurali.

\section{INDICE}

Indice geografico : Timgad

\section{AUTORI}

\section{FRANCESCO MARTORELLA}

Università degli Studi della Basilicata - francesco.martorella@gmail.com

\section{FATIMA-ZOHRA BAHLOUL}

Université de Batna 1 - f-zbahloul@hotmail.fr 